\title{
Mode visibilities in rapidly rotating stars ${ }^{\star}$
}

\author{
D. R. Reese ${ }^{1,2,3}$, V. Prat ${ }^{4,5}$, C. Barban ${ }^{2}$, C. van 't Veer-Menneret ${ }^{6}$, and K. B. MacGregor ${ }^{7}$
}

\author{
${ }^{1}$ Institut d'Astrophysique et Géophysique de l'Université de Liège, Allée du 6 Août 17, 4000 Liège, Belgium \\ e-mail: daniel.reese@ulg.ac.be \\ 2 LESIA, Observatoire de Paris, CNRS, UPMC Univ. Paris 06, Univ. Paris-Diderot, 5 place Jules Janssen, 92195 Meudon, France \\ 3 Kavli Institute for Theoretical Physics, Kohn Hall, University of California, Santa Barbara, CA 93106, USA \\ ${ }^{4}$ Universté de Toulouse, UPS-OMP, IRAP, 31400 Toulouse, France \\ 5 CNRS, IRAP, 14 avenue Édouard Belin, 31400 Toulouse, France \\ ${ }^{6}$ GEPI, Observatoire de Paris-Meudon, CNRS, Université Paris Diderot, 92125 Meudon Cedex, France \\ 7 High Altitude Observatory, National Center for Atmospheric Research, Boulder, CO 80307, USA
}

Received 5 October 2012 / Accepted 5 December 2012

\begin{abstract}
Context. Mode identification is a crucial step to comparing observed frequencies with theoretical ones. However, it has proven to be particularly difficult in rapidly rotating stars. An important reason for this is the lack of simple frequency patterns such as those present in solar-type pulsators. This problem is further aggravated in $\delta$ Scuti stars by their particularly rich frequency spectra.

Aims. As a first step to obtaining further observational constraints towards mode identification in rapid rotators, we aim to accurately calculate mode visibilities and amplitude ratios while fully taking into account the effects of rotation.

Methods. We derive the relevant equations for calculating mode visibilities in different photometric bands while fully taking into account the geometric distortion from both the centrifugal deformation and the pulsation modes, the variations in effective gravity, and an approximate treatment of the temperature variations, given the adiabatic nature of the pulsation modes. These equations are then applied to 2D oscillation modes, calculated using the TOP code (Two-dimension Oscillation Program), in fully distorted 2D models based on the self-consistent field (SCF) method. The specific intensities come from a grid of Kurucz atmospheres, thereby taking into account limb and gravity darkening.

Results. We obtain mode visibilities and amplitude ratios for $2 M_{\odot}$ models with rotation rates ranging from 0 to $80 \%$ of the critical rotation rate. Based on these calculations, we confirm a number of results from earlier studies, such as the increased visibility of numerous chaotic modes at sufficient rotation rates, the simpler frequency spectra with dominant island modes for pole-on configurations, or the dependence of amplitude ratios on inclination and azimuthal order in rotating stars. In addition, we explain how the geometric shape of the star leads to a smaller contrast between pole-on and equator-on visibilities of equatorially-focused island modes. We also show that modes with similar $(\ell,|m|)$ values frequently have similar amplitude ratios, even in the most rapidly rotating models.
\end{abstract}

Key words. stars: oscillations - stars: rotation - stars: interiors - stars: variables: $\delta$ Scuti

\section{Introduction}

The space missions CoRoT (Baglin et al. 2009; Auvergne et al. 2009) and Kepler (Borucki et al. 2009) are revealing very rich pulsational spectra in rapidly rotating $\delta$ Scuti stars. For instance, several hundred individual frequencies have been found in HD 50844 and HD 181555, observed by CoRoT (Poretti et al. 2009; Michel, priv. comm.), and V2367 Cyg, observed by Kepler (Balona et al. 2012). It is becoming increasingly clear that interpreting these spectra will not be a straightforward task and that theory is lagging behind observations. A crucial first step in interpreting this data is correctly identifying the pulsation modes, i.e. finding the correct correspondence between theoretically calculated modes and observed pulsations. Recently, Reese et al. (2009b) proposed a way to identify acoustic pulsation modes in rapidly rotating stars based on an asymptotic formula which describes the frequencies of low degree modes (see Pasek et al. 2012, and references therein). Nonetheless this method runs into trouble if chaotic modes are present in the pulsation spectra, which is expected based on the visibility calculations in Lignières \& Georgeot (2009). Furthermore, the pulsation modes in $\delta$ Scuti stars tend to be of low radial order, and may therefore be too far from the asymptotic regime. Lignières et al. (2010) have worked on using the cross-correlation of pulsation spectra. Although it doesn't yield individual mode identifications, it may provide a way of obtaining the rotation rate and/or the large frequency separation, and explaining recent observations of recurring frequency spacings in rapid rotators (García Hernández et al. 2009; Mantegazza et al. 2012). Nonetheless, the need remains for methods capable of identifying individual pulsation modes.

Two particularly promising methods for identifying pulsation modes are multi-colour photometric and spectroscopic mode identification. The first approach consists in measuring the amplitudes and phases of a given pulsation mode in different photometric

\footnotetext{
* Appendices are available in electronic form at http://www. aanda.org
} 
bands, calculating the ratios of the different amplitudes and/or the phase differences, and comparing these to theoretical predictions. The second approach exploits the Doppler shifts caused by the velocity field from the pulsation mode and how it affects observed absorption lines. These methods have been successfully applied to slowly rotating stars (e.g. De Ridder et al. 2004; Zima et al. 2006; Briquet et al. 2007), but more work is needed before they are applied to rapid rotators. In the present paper, we will focus on mode visibilities in different photometric bands as a first step to multi-colour photometric mode identification, and postpone spectroscopic mode identification to a later paper.

Few studies have dealt with multi-colour photometric mode signatures in rapidly rotating stars, and those that do generally approximate the effects of rotation on the pulsation modes. For instance, Daszyńska-Daszkiewicz et al. (2002, 2007) and Townsend (2003a) used either the perturbative approach or the traditional approximation to calculate their pulsation modes, and in some cases included the effects of avoided crossings. Their calculations of mode visibilities included stellar surface distortion from the pulsation modes and the Lagrangian perturbations to both the effective temperature and gravity. An interesting result from these studies is that contrarily to non-rotating stars, amplitude ratios depend both on $m$, the azimuthal order of the pulsation mode, and $i$, the inclination of the star. More recently, Lignières et al. (2006) and Lignières \& Georgeot (2009) calculated geometrical diskintegration factors of acoustic modes in deformed polytropic models by integrating the temperature fluctuations over the visible disk. The effects of rotation were fully taken into account in the pulsation modes, thanks to the 2D numerical approach, but nonadiabatic effects were neglected, thereby making the fluctuations of the effective temperature inaccessible. Furthermore, gravity and limb darkening, the Lagrangian perturbations to the effective gravity, and surface distortion caused by the modes were not taken into account. Nonetheless, important first results were obtained through these articles, namely, that chaotic modes are more visible than their non-rotating counterparts due to irregular latitudinal node placement and may thus be detected, island modes are the most visible modes in a pole-on configuration, leading to a regular frequency pattern (Lignières \& Georgeot 2009), and signatures of the large frequency separation and/or rotation rate can show up in the autocorrelation function of the frequency spectrum (Lignières et al. 2010).

In order to obtain more realistic multi-colour photometric mode visibilities in rapidly rotating stars, we derive a new set of equations which take into account the Lagrangian variations to the effective temperature and gravity, as well as the surface distortions induced both by the centrifugal deformation of the equilibrium model and by the pulsation modes. These are applied to adiabatic acoustic modes calculated by the Two-dimension Oscillation Program (TOP, Reese et al. 2006, 2009a) using rapidly rotating zeroage main-sequence (ZAMS) models based on the self-consistent field (SCF) method (Jackson et al. 2005; MacGregor et al. 2007). The emergent intensities are calculated from Kurucz atmospheres, taking into account the latitudinal dependence of the equilibrium effective temperature and gravity, thereby including gravity and limb darkening. The main weakness in the present study is the adiabatic approximation, which makes the Lagrangian fluctuations of the effective temperature inaccessible. As was previously done in Lignières \& Georgeot (2009), we approximate these by the Lagrangian temperature variations. The following section describes the pulsation calculations, with an emphasis on the improvements and differences with the calculations done in Reese et al. (2009a). This section is followed by a derivation of the relevant equations for calculating mode visibilities in rapidly rotating stars. Section 4 then describes various effects of rotation on visibilities in a single band - the CoRoT photometric band. This is followed by Sect. 5 which deals with amplitude ratios in the Geneva photometric system. The paper ends with a short conclusion.

\section{Pulsation calculations}

In what follows, we review the methods used for obtaining the models and associated pulsations that serve as inputs to the visibility calculations. These closely follow the approach used in Reese et al. (2009a) but include a number of improvements as described below.

\subsection{Equilibrium models}

The equilibrium models are calculated via the SCF method (Jackson et al. 2005; MacGregor et al. 2007). This method is an iterative procedure which alternates between solving Poisson's equation and the equations of mass, momentum and energy conservation before converging onto a 2D centrifugally deformed stellar model. These models are chemically homogeneous ZAMS models with a cylindrical rotation profile, although throughout the rest of the article, we will work with uniformly rotating SCF models, even if the formulas in the visibility calculations are established for general (non-cylindrical) rotation profiles. Given the rotation profile, the structure of the model is barotropic, i.e. all thermodynamic quantities remain constant on isopotentials, which are calculated from the sum of the gravitational and centrifugal potentials. Finally, we wish to make the distinction between the critical rotation rate, $\Omega_{\mathrm{C}}$, and the Keplerian break-up rotation rate, $\Omega_{\mathrm{K}}$ :

$\Omega_{\mathrm{C}}=\sqrt{\frac{g_{\mathrm{eq}}}{R_{\mathrm{eq}}}}, \quad \Omega_{\mathrm{K}}=\sqrt{\frac{G M}{R_{\mathrm{eq}}^{3}}}$.

Although very similar, the former uses the true gravity (excluding the centrifugal force) at the equator, $g_{\text {eq }}$, based on the actual distribution of matter, to calculate the break-up rotation rate, whereas the latter uses its Keplerian approximation, $\frac{G M}{R_{\mathrm{eq}}^{2}}$, which amounts to assuming spherical symmetry for the distribution of matter. As pointed out in Roxburgh (2004), the Keplerian approximation slightly underestimates the true gravity, so that $\Omega_{\mathrm{C}}>\Omega_{\mathrm{K}}$. Table 1 gives the relative differences between these two quantities for different rotation rates, calculated two different ways. The first method is based on global quantities provided with the models, whereas the second involves recalculating the gravitational potential from the density distribution and using this to calculate the equatorial gravity. A comparison of columns two and three, and five and six gives an idea of the uncertainty on these values. We 
D. R. Reese et al.: Mode visibilities in rapidly rotating stars

Table 1. Relative differences, $\left(\Omega_{\mathrm{C}}-\Omega_{\mathrm{K}}\right) / \Omega_{\mathrm{K}}$, for selected rotation rates, calculated with two different methods (see text for details).

\begin{tabular}{lccccc}
\hline \hline$\Omega_{\mathrm{C}}$ & Method 1 & Method 2 & $\Omega_{\mathrm{C}}$ & Method 1 & Method 2 \\
\hline 0.00 & - & $1.1 \times 10^{-4}$ & 0.50 & $5.0 \times 10^{-4}$ & $4.7 \times 10^{-4}$ \\
0.10 & $9.5 \times 10^{-5}$ & $4.9 \times 10^{-5}$ & 0.60 & $5.7 \times 10^{-4}$ & $5.5 \times 10^{-4}$ \\
0.20 & $2.3 \times 10^{-4}$ & $2.1 \times 10^{-4}$ & 0.70 & $6.0 \times 10^{-4}$ & $5.8 \times 10^{-4}$ \\
0.30 & $3.0 \times 10^{-4}$ & $2.7 \times 10^{-4}$ & 0.80 & $4.9 \times 10^{-4}$ & $4.4 \times 10^{-4}$ \\
0.40 & $3.1 \times 10^{-4}$ & $2.6 \times 10^{-4}$ & & & \\
\hline
\end{tabular}

also note that the theoretical value of $\left(\Omega_{\mathrm{C}}-\Omega_{\mathrm{K}}\right) / \Omega_{\mathrm{K}}$ at $\Omega_{\mathrm{C}}=0.00$ is 0 , since the star is spherically symmetric and the Keplerian approximation is exact.

Before being used in the pulsation calculations, the models need to be interpolated onto a new grid, and a number of supplementary equilibrium quantities have to be derived, including a variety of geometric terms as well as gradients of different equilibrium quantities. Since Reese et al. (2009a), a number of improvements have been incorporated into these procedures. For instance, the stellar models are now interpolated onto a non-uniform radial grid which becomes dense near the stellar surface. This allows the pulsation code to correctly resolve the rapid spatial variations of acoustic modes near the surface, resulting from the decrease in sound velocity. Instead of interpolating the density and pressure directly, their logarithm is interpolated. This leads to more accurate and consistent values near the surface, where these quantities are several orders of magnitude smaller than in the centre, and ensures they remain positive. The effective gravity is calculated via Poisson's equation. This avoids taking the ratio of the pressure gradient divided by the density, both of which are small quantities subject to relatively large uncertainties. Furthermore, the equipotentials, and hence the geometric structure of the star, are recalculated using the solution from Poisson's equation thus removing some numerical inaccuracies in the original models. The $\zeta$ derivative of equilibrium quantities is now correctly calculated. In Reese et al. (2009a), the derivative was mistakenly calculated with respect to $r$ rather than $\zeta$. Although this changed the quantitative results, the qualitative conclusions from Reese et al. (2009a) remain unaltered. Finally, the $\Gamma_{1}$ profile is not derived from Eq. (16) of Jackson et al. (2005), but rather from the equation of state, which is based on the formula of Eggleton et al. (1973). Furthermore, the quantity $\Gamma_{2}$, which intervenes in the visibility calculations described below, is also calculated via the equation of state.

\subsection{Pulsation equations}

A new set of variables is used in the pulsation equations:

$\xi, \quad \frac{\delta p}{P_{0}}, \quad \frac{\delta \rho}{\rho_{0}}, \quad \Psi$,

where $\xi$ is the Lagrangian displacement, $\delta p / P_{0}$ the Lagrangian pressure perturbation divided by the equilibrium pressure profile, $\delta \rho / \rho_{0}$ the Lagrangian density perturbation divided by the equilibrium density profile, and $\Psi$ the Eulerian perturbation to the gravitational potential. Throughout this article, the subscript " 0 " denotes equilibrium quantities. We assume that the time and $\phi$ dependence of these variables takes on the form $\mathrm{e}^{\mathrm{i}(\omega t+m \phi)}$, where $m$ is the azimuthal order. As such, we use what could be called the "retrograde convention", i.e. modes with positive azimuthal orders, $m$, are retrograde.

Based on these variables, the continuity equation becomes

$0=\frac{\delta \rho}{\rho_{0}}+\boldsymbol{\nabla} \cdot \boldsymbol{\xi}$

and Poisson's equation is

$0=\Delta \Psi-4 \pi G\left(\rho_{0} \frac{\delta \rho}{\rho_{0}}-\boldsymbol{\xi} \cdot \nabla \rho_{0}\right)$,

where $G$ is the gravitational constant. Euler's equation takes some more manipulations (see, for example, Eq. (A.3) of Reese et al. 2009a):

$$
\begin{aligned}
-[\omega+m \Omega]^{2} \boldsymbol{\xi} & +2 i[\omega+m \Omega] \boldsymbol{\Omega} \times \boldsymbol{\xi}+\xi_{s} s \partial_{s}\left(\Omega^{2}\right) \boldsymbol{e}_{s}=-\frac{\boldsymbol{\nabla} p}{\rho_{0}}+\frac{\rho \boldsymbol{g}_{\mathrm{eff}}}{\rho_{0}}-\boldsymbol{\nabla} \Psi \\
& =-\frac{1}{\rho_{0}} \boldsymbol{\nabla}\left(P_{0} \frac{\delta p}{P_{0}}-\boldsymbol{\xi} \cdot \boldsymbol{\nabla} P_{0}\right)+\left(\rho_{0} \frac{\delta \rho}{\rho_{0}}-\boldsymbol{\xi} \cdot \boldsymbol{\nabla} \rho_{0}\right) \frac{\boldsymbol{\nabla} P_{0}}{\rho_{0}^{2}}-\boldsymbol{\nabla} \Psi \\
& =-\frac{P_{0}}{\rho_{0}} \boldsymbol{\nabla}\left(\frac{\delta p}{P_{0}}\right)-\frac{\delta p}{P_{0}} \frac{\boldsymbol{\nabla} P_{0}}{\rho_{0}}+\frac{\boldsymbol{\nabla}\left(\boldsymbol{\xi} \cdot \boldsymbol{\nabla} P_{0}\right)}{\rho_{0}}+\frac{\delta \rho}{\rho_{0}} \frac{\boldsymbol{\nabla} P_{0}}{\rho_{0}}-\frac{\left(\boldsymbol{\xi} \cdot \boldsymbol{\nabla} \rho_{0}\right) \boldsymbol{\nabla} P_{0}}{\rho_{0}^{2}}-\boldsymbol{\nabla} \Psi \\
& =-\frac{P_{0}}{\rho_{0}} \boldsymbol{\nabla}\left(\frac{\delta p}{P_{0}}\right)+\left(\frac{\delta \rho}{\rho_{0}}-\frac{\delta p}{P_{0}}\right) \boldsymbol{g}_{\mathrm{eff}}+\boldsymbol{\nabla}\left(\boldsymbol{\xi} \cdot \boldsymbol{g}_{\mathrm{eff}}\right)-\boldsymbol{\nabla} \Psi+\left\{\frac{\left(\boldsymbol{\xi} \cdot \boldsymbol{\nabla} P_{0}\right) \boldsymbol{\nabla} \rho_{0}-\left(\boldsymbol{\xi} \cdot \boldsymbol{\nabla} \rho_{0}\right) \boldsymbol{\nabla} P_{0}}{\rho_{0}^{2}}\right\}
\end{aligned}
$$


where $\Omega$ is the rotation profile, $s$ the distance to the rotation axis, and $\boldsymbol{g}_{\text {eff }}=-\nabla \Psi_{0}+s \Omega^{2} \boldsymbol{e}_{s}=\nabla P_{0} / \rho_{0}$ the effective gravity. The term in curly brackets cancels out because $\nabla P_{0}$ is parallel to $\nabla \rho_{0}$ in a barotropic stellar structure. Finally, the adiabatic relation takes on the following very simple form:

$\frac{\delta \rho}{\rho_{0}}=\frac{1}{\Gamma_{1}} \frac{\delta p}{P_{0}}$

This last equation is then used to eliminate $\delta \rho / \rho_{0}$ in favour of $\delta p / P_{0}$ throughout the differential system and thus to reduce the size of the problem compared to what is obtained in Reese et al. (2009a). Explicit expressions for Eqs. (3), (4) and (5), using spheroidal coordinates (see Sect. 2.4), are given in Appendix A.1.

Besides reducing the computational cost, using the variables in Eq. (2) allows us to obtain a much cleaner derivation of $\delta p / P_{0}$, and hence $\delta T / T_{0}$, near the surface. Indeed, if one were to calculate this quantity from the Eulerian pressure perturbation, they would apply the following relation:

$\frac{\delta p}{P_{0}}=\frac{p+\boldsymbol{\xi} \cdot \boldsymbol{\nabla} P_{0}}{P_{0}}$

Near the surface, this involves the sum of two nearly opposite terms, divided by a small quantity, thus leading to poor numerical results.

\subsection{Non-dimensionalisation}

Contrarily to Reese et al. (2009a), we non-dimensionalise the SCF models in a more classical way. The following reference quantities are used as units of length, density and pressure:

$R_{\mathrm{eq}}, \quad \rho_{\text {ref }}=\frac{M}{R_{\mathrm{eq}}^{3}}, \quad P_{\text {ref }}=\frac{G M^{2}}{R_{\mathrm{eq}}^{4}}$,

where $R_{\mathrm{eq}}$ is the equatorial radius and $M$ the mass. These lead to the following time scale:

$t_{\mathrm{ref}}=\left(\frac{R_{\mathrm{eq}}^{3}}{G M}\right)^{1 / 2}=\frac{1}{\Omega_{\mathrm{K}}}$,

where $\Omega_{\mathrm{K}}$ is the Keplerian break-up rotation rate. Hence, the non-dimensional frequencies are directly $\omega / \Omega_{\mathrm{K}}$. With this non-dimensionalisation, the preceding pulsation equations remain unchanged except for Poisson's equation, which becomes

$0=\Delta \Psi-4 \pi\left(\rho_{0} \frac{\delta \rho}{\rho_{0}}-\boldsymbol{\xi} \cdot \nabla \rho_{0}\right)$.

\subsection{Spheroidal geometry}

As was done in Lignières et al. (2006) and Reese et al. (2006), a surface-fitting coordinate system, $(\zeta, \theta, \phi)$, based on Bonazzola et al. (1998), is introduced. This system is related to the usual spherical coordinates, $(r, \theta, \phi)$, via the relation

$r(\zeta, \theta)=(1-\varepsilon) \zeta+\frac{5 \zeta^{3}-3 \zeta^{5}}{2}\left(R_{s}(\theta)-1+\varepsilon\right)$,

where $R_{S}(\theta)$ corresponds to the surface and $\varepsilon=1-R_{\mathrm{p}} / R_{\mathrm{eq}}$ is a measure of the oblateness, $R_{\mathrm{p}}$ being the polar radius, and $\zeta$ is comprised between 0 and 1 . As can be seen, $\zeta=1$ corresponds to the stellar surface. A second domain, with a spherical outer boundary, is added around the star so as to simplify the boundary condition on the gravitational potential:

$r(\zeta, \theta)=2 \varepsilon+(1-\varepsilon) \zeta+\left(2 \zeta^{3}-9 \zeta^{2}+12 \zeta-4\right)\left(R_{s}(\theta)-1-\varepsilon\right)$,

where $\zeta \in[1,2]$. For $\zeta=1$, Eq. (11) coincides with the stellar surface, whereas for $\zeta=2$, it yields a sphere of radius 2 (or $2 R_{\mathrm{eq}}$ in dimensional form). For conciseness, we will use the subscripts " $\zeta$ " and " $\theta$ " to denote derivatives of $r$ with respect to these variables. For example, $r_{\zeta}=\partial r / \partial \zeta$ and $r_{\zeta \theta}=\partial^{2} r / \partial \zeta \partial \theta$.

\subsection{Boundary conditions}

The pulsation equations are supplemented by a number of boundary equations. Regularity of the solutions is imposed in the centre. The perturbation to the gravitational potential is made to go to zero at an infinite distance from the star. This condition is imposed by extending $\Psi$ into the second domain and matching it to a vacuum potential on the second domain's outer spherical boundary as described in Reese et al. (2006). When extending $\Psi$ into the second domain, both it and its gradient need to be kept continuous across the perturbed stellar surface. This can be achieved by imposing the continuity of the Lagrangian perturbation to the gravitational potential and its gradient at $\zeta=1$ :

$\Psi^{\mathrm{int}}+\boldsymbol{\xi} \cdot \boldsymbol{\nabla} \Psi_{0}^{\mathrm{int}}=\Psi^{\mathrm{ext}}+\boldsymbol{\xi} \cdot \nabla \Psi_{0}^{\mathrm{ext}}, \quad \nabla \Psi^{\mathrm{int}}+\boldsymbol{\xi} \cdot \boldsymbol{\nabla}\left(\boldsymbol{\nabla} \Psi_{0}^{\mathrm{int}}\right)=\boldsymbol{\nabla} \Psi^{\mathrm{ext}}+\boldsymbol{\xi} \cdot \boldsymbol{\nabla}\left(\boldsymbol{\nabla} \Psi_{0}^{\mathrm{ext}}\right)$,

A77, page 4 of 28 
D. R. Reese et al.: Mode visibilities in rapidly rotating stars
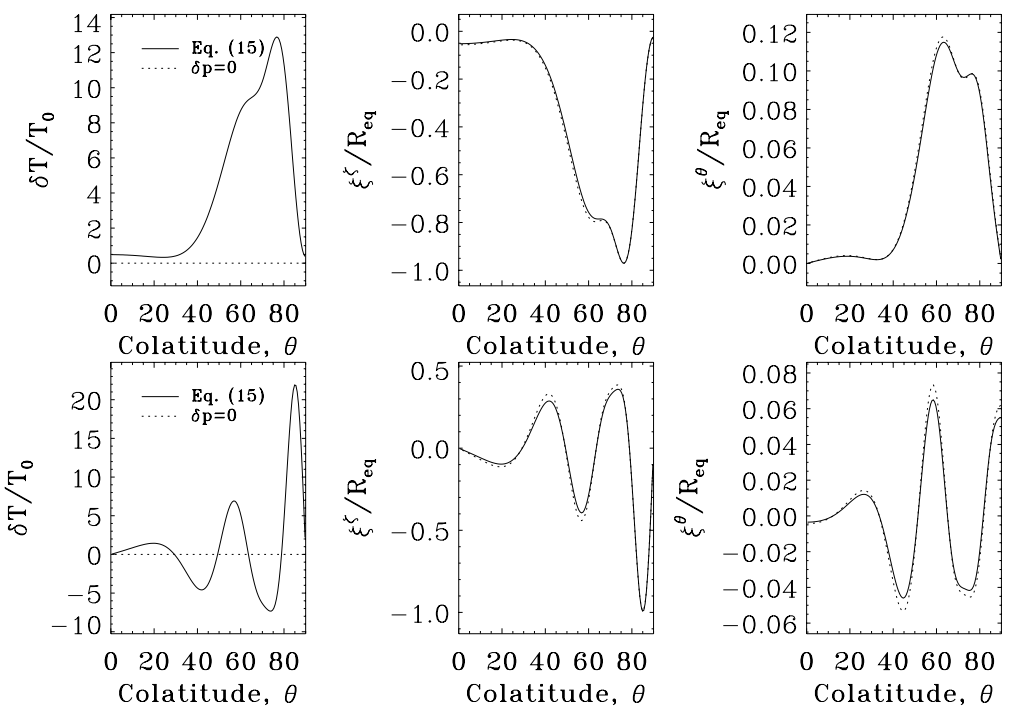

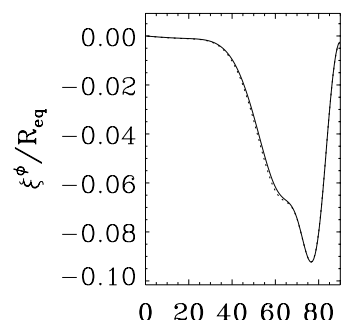

020406080
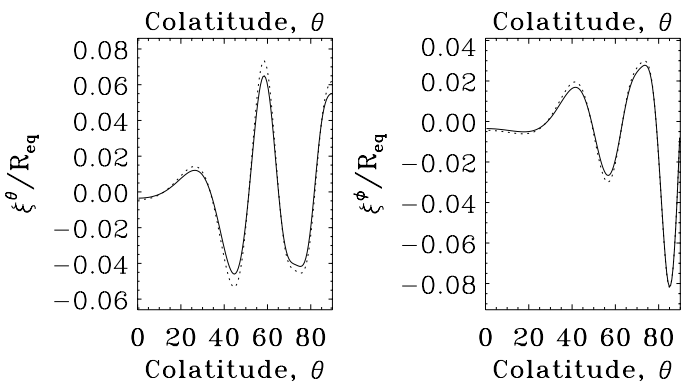

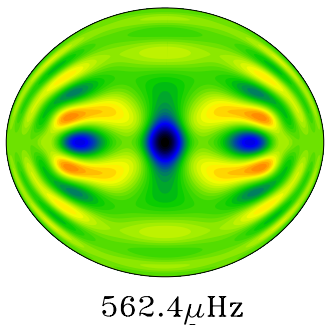

$\mathrm{m}=0$

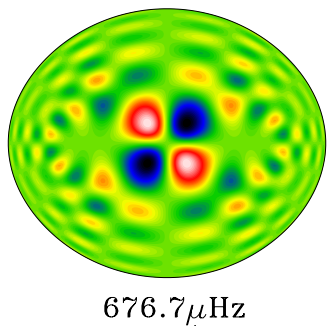

$\mathrm{m}=1$

Fig. 1. Lagrangian temperature variations and Lagrangian displacement at the stellar surface for two modes, using either $\delta p=0$ or Eq. (15) as an external boundary condition. The figures to the right display the meridional cross-section of the Eulerian pressure perturbation of the two modes, divided by the square-root of the equilibrium density.

where the superscripts "int" and "ext" correspond to "just below" and "just above" the stellar surface, respectively. Given that the gradient of the equilibrium gravitational potential is continuous, the first condition simplifies to

$\Psi^{\text {int }}=\Psi^{\text {ext }}$.

The second condition can be simplified, using Poisson's equation, applied to the equilibrium gravitational potential:

$\partial_{\zeta} \Psi^{\mathrm{int}}=\partial_{\zeta} \Psi^{\mathrm{ext}}-\frac{4 \pi G \rho_{0} \zeta^{2} r_{\zeta}}{r^{2}+r_{\theta}^{2}} \xi^{\zeta}, \quad \partial_{\theta} \Psi^{\mathrm{int}}=\partial_{\theta} \Psi^{\mathrm{ext}}, \partial_{\phi} \Psi^{\mathrm{int}}=\partial_{\phi} \Psi^{\mathrm{ext}}$

Here, $\xi^{\zeta}$ is the radial component of the Lagrangian displacement, when decomposed over the alternate basis $\left(\boldsymbol{a}_{\zeta}, \boldsymbol{a}_{\theta}, \boldsymbol{a}_{\phi}\right)$ introduced in Reese et al. (2006, see also Eq. (A.3)). The first equation is different from what was applied in Reese et al. (2009a), since it takes into account the contribution from a non-zero surface density. However, given its low value, the resultant difference is quite negligible. The latter two equations are implied by Eq. (13), making it unnecessary to impose them.

Finally, the usual mechanical boundary condition on the Lagrangian pressure perturbation, $\delta \mathbf{p}=\mathbf{0}$, has been replaced by a slightly different condition:

$\boldsymbol{E}^{\zeta} \cdot \boldsymbol{\nabla}\left(\frac{\delta p}{P_{0}}\right)=0$,

where $\boldsymbol{E}^{\zeta}$ is a vector perpendicular to the surface (see Eq. (A.2)). The above, modified mechanical condition corresponds to setting the vertical gradient of $\delta p / P_{0}$, rather than its value, to zero. We note that Pesnell (1990) and Dupret et al. (2002) applied a similar condition for spherically symmetric stars. In order to avoid having a boundary condition with a radial derivative in it, we calculate the dot product between Euler's equation and $\boldsymbol{E}^{\zeta}$ and cancel out the term corresponding to the vertical gradient of $\delta p / P_{0}$. This leads to a complicated expression which is given in spheroidal coordinates in Appendix A.2.

The main purpose in using Eq. (15) is to obtain a non-zero value for $\delta T / T_{0}$, useful for visibility calculations as described below. Indeed, when combined with a non-zero surface pressure and the adiabatic relation, the simpler condition, $\delta p=0$, leads to $\delta T / T_{0}=0$, as illustrated in Fig. 1 (left panels). One may then wonder if Eq. (15) has an important effect on the frequencies and on the displacement at the surface. Numerically, it turns out the frequencies vary little when using either boundary condition, at least in the present study. The middle panels of Fig. 1 also show that the displacement is hardly affected.

\subsection{Numerical method}

This set of equations and boundary conditions is projected onto the spherical harmonic basis and discretised in the radial direction before being solved using the code TOP (Reese et al. 2006, 2009a). Besides the improved way of treating the equilibrium model, the present calculations also benefit from a new form of finite differences. This form achieves 4 th order accuracy for 1 st order derivatives in spite of using windows with 4 rather 5 grid points. More importantly, this approach is robust to problems like mesh drift and spurious solutions. 


\section{Mode visibilities}

At this point, we will switch to using the spherical vector basis $\left(\boldsymbol{e}_{r}, \boldsymbol{e}_{\theta}, \boldsymbol{e}_{\phi}\right)$ in which the polar or z-axis is lined up with the star's rotation axis. Furthermore, in order to make the equations more compact, we will prefer the notation " $r$ " to " $R_{\mathrm{s}}$ " when designating the stellar surface, although it should be understood as $r(\zeta=1, \theta)$ in what follows. Similar implicit arguments also apply to other geometric terms such as $r_{\zeta}$ and $r_{\theta}$. We introduce the unit vector $\boldsymbol{e}_{\mathrm{obs}}$. which points from the star to the observer. Furthermore, we will assume that the vector $\boldsymbol{e}_{\mathrm{obs}}$ lies in the meridional plane $\phi=0$. Let $i$ be the inclination angle, i.e. the angle between $\boldsymbol{e}_{z}$ and $\boldsymbol{e}_{\mathrm{obs}}$, where $\boldsymbol{e}_{z}$ is lined up with the rotation axis ${ }^{1}$. An explicit expression for $\boldsymbol{e}_{\mathrm{obs}}$ in terms of the usual spherical basis is:

$\boldsymbol{e}_{\mathrm{obs} .}=(\sin i \sin \theta \cos \phi+\cos i \cos \theta) \boldsymbol{e}_{r}+(\sin i \cos \theta \cos \phi-\cos i \sin \theta) \boldsymbol{e}_{\theta}-\sin i \sin \phi \boldsymbol{e}_{\phi}$

The radiated energy, received by an observing instrument from a non-pulsating stars, is:

$E=\frac{1}{2 \pi d^{2}} \iint_{\text {Vis.Surf. }} I\left(\mu, g_{\text {eff }}, T_{\text {eff }}\right) \boldsymbol{e}_{\text {obs. }} \cdot \mathrm{d} \boldsymbol{S}$

where $d$ is the distance to the star, $\mu$ the cosine of the angle between the outward normal to the surface and $\boldsymbol{e}_{\mathrm{obs}} . g_{\mathrm{eff}}$ and $T_{\mathrm{eff}}$ the effective gravity and temperature, and $I\left(\mu, g_{\text {eff }}, T_{\text {eff }}\right)$ the specific radiation intensity, multiplied by the instrument's and/or filter's transmission curve, and integrated over the wavelength spectrum,. As can be seen, the integral is carried out over the visible surface.

In a pulsating star, this quantity is perturbed as follows:

$$
\Delta E(t)=\frac{1}{2 \pi d^{2}} \mathfrak{R}\left\{\iint_{\Delta \text { (Vis.Surf. })} I\left(\mu, g_{\text {eff }}, T_{\text {eff }}\right) \boldsymbol{e}_{\mathrm{obs} .} \cdot \mathrm{d} \boldsymbol{S}+\iint_{\text {Vis.Surf. }} \delta I\left(\mu, g_{\mathrm{eff}}, T_{\mathrm{eff}}, t\right) \boldsymbol{e}_{\mathrm{obs} .} \cdot \mathrm{d} \boldsymbol{S}+\iint_{\text {Vis.Surf. }} I\left(\mu, g_{\mathrm{eff}}, T_{\mathrm{eff}}\right) \boldsymbol{e}_{\mathrm{obs} .} \cdot \delta(\mathrm{d} \boldsymbol{S})\right\}
$$

where $\delta$ denotes the Lagrangian perturbation, $\mathfrak{R}\{\ldots\}$ the real part, and $\Delta$ (Vis.Surf.) the amount by which the visible surface is modified due the modifications of the surface normal induced by the oscillatory motions. Furthermore, we assume a complex form for the eigenfunctions, hence the reason for taking the real part of the above expression.

The first term in Eq. (18) is proportional to the square of the displacement and therefore neglected (e.g. Dziembowski 1977).

The Lagrangian perturbation to the specific intensity which intervenes in the second term may be developed as follows:

$\delta I=I\left(\frac{\partial \ln I}{\partial \ln T_{\mathrm{eff}}} \frac{\delta T_{\mathrm{eff}}}{T_{\mathrm{eff}}}+\frac{\partial \ln I}{\partial \ln g_{\mathrm{eff}}} \frac{\delta g_{\mathrm{eff}}}{g_{\mathrm{eff}}}\right)+\frac{\partial I}{\partial \mu} \delta \mu$.

The partial derivatives of $I$ are obtained from model atmospheres and will be dealt with in Sect. 3.4. We note that the above expression is not exact as it neglects the slight Doppler shifts caused by rotation and the oscillations. Such shifts modify the position of the emerging flux with respect to the instrument's and/or filter's transmission curve thereby modifying $I$, but are expected to play a negligible role compared to other effects. We are therefore left with a number of geometrical terms to calculate as well as the Lagrangian perturbation to the effective temperature and gravity.

\subsection{Geometrical terms}

In what follows, we will use the following expression for the displacement:

$\boldsymbol{\xi}=\xi_{r}(\zeta, \theta, \phi, t) \boldsymbol{e}_{r}(\theta, \phi)+\xi_{\theta}(\zeta, \theta, \phi, t) \boldsymbol{e}_{\theta}(\theta, \phi)+\xi_{\phi}(\zeta, \theta, \phi, t) \boldsymbol{e}_{\phi}(\phi)$

where $\left(\boldsymbol{e}_{r}, \boldsymbol{e}_{\theta}, \boldsymbol{e}_{\phi}\right)$ is the usual vector basis in spherical coordinates. Note: we have used subscripts rather than superscripts, for the letters $r, \theta$, and $\phi$, to distinguish these components from those given in Eq. (A.4). In what follows, we will be using the following relations:

$\begin{array}{llll}\partial_{\theta} \boldsymbol{e}_{r} & =\boldsymbol{e}_{\theta}, & \partial_{\theta} \boldsymbol{e}_{\theta}=-\boldsymbol{e}_{r}, & \partial_{\theta} \boldsymbol{e}_{\phi}=\mathbf{0}, \\ \partial_{\phi} \boldsymbol{e}_{r}=\sin \theta \boldsymbol{e}_{\phi}, & \partial_{\phi} \boldsymbol{e}_{\theta}=\cos \theta \boldsymbol{e}_{\phi}, & \partial_{\phi} \boldsymbol{e}_{\phi}=-\sin \theta \boldsymbol{e}_{r}-\cos \theta \boldsymbol{e}_{\theta} .\end{array}$

We start by calculating a surface element on a rotating, non-pulsating star. This is given by the following expression:

$\mathrm{d} \boldsymbol{S}=\left(\partial_{\theta} \boldsymbol{r} \times \partial_{\phi} \boldsymbol{r}\right) \mathrm{d} \theta \mathrm{d} \phi=\left(r^{2} \sin \theta \boldsymbol{e}_{r}-r r_{\theta} \sin \theta \boldsymbol{e}_{\theta}\right) \mathrm{d} \theta \mathrm{d} \phi$.

We then calculate the Lagrangian perturbation to a surface element:

$$
\begin{aligned}
\delta(\mathrm{d} \boldsymbol{S})= & \left(\partial_{\theta} \boldsymbol{\xi} \times \partial_{\phi} \boldsymbol{r}+\partial_{\theta} \boldsymbol{r} \times \partial_{\phi} \boldsymbol{\xi}\right) \mathrm{d} \theta \mathrm{d} \phi \\
= & \left\{\left(2 r \sin \theta \xi_{r}+r \cos \theta \xi_{\theta}+r \sin \theta \partial_{\theta} \xi_{\theta}+r \partial_{\phi} \xi_{\phi}\right) \boldsymbol{e}_{r}+\left(-r_{\theta} \sin \theta \xi_{r}-r \sin \theta \partial_{\theta} \xi_{r}+\left(r \sin \theta-r_{\theta} \cos \theta\right) \xi_{\theta}-r_{\theta} \partial_{\phi} \xi_{\phi}\right) \boldsymbol{e}_{\theta}\right. \\
& \left.+\left(-r \partial_{\phi} \xi_{r}+r_{\theta} \partial_{\phi} \xi_{\theta}+\left(r \sin \theta-r_{\theta} \cos \theta\right) \xi_{\phi}\right) \boldsymbol{e}_{\phi}\right\} \mathrm{d} \theta \mathrm{d} \phi .
\end{aligned}
$$

1 As opposed to a non-rotating star, the inclination cannot be arbitrarily set to 0 to simplify the calculations. 
The approach used to obtain Eqs. (22) and (23) is essentially the same as that of Buta \& Smith (1979) and Townsend (1997), but the effects of horizontal Lagrangian displacements are also included in the latter equation. We then calculate $\mu$ :

$\mu=\boldsymbol{e}_{\mathrm{obs} .} \cdot \boldsymbol{n}=\frac{\left[r(\sin i \sin \theta \cos \phi+\cos i \cos \theta)-r_{\theta}(\sin i \cos \theta \cos \phi-\cos i \sin \theta)\right]}{\left[r^{2}+r_{\theta}^{2}\right]^{1 / 2}}$,

where $\boldsymbol{n}=\frac{\mathrm{d} \boldsymbol{S}}{\|\mathrm{d} \boldsymbol{S}\|}$. The Lagrangian perturbation to $\mu$ is calculated as follows:

$$
\begin{aligned}
\delta \mu= & \boldsymbol{e}_{\text {obs. }} \cdot \delta \boldsymbol{n}=\boldsymbol{e}_{\mathrm{obs} .} \cdot\left\{\frac{\delta \mathrm{d} \boldsymbol{S}}{\|\mathrm{d} \boldsymbol{S}\|}-\frac{\mathrm{d} \boldsymbol{S}}{\|\mathrm{d} \boldsymbol{S}\|^{2}} \delta\|\mathrm{d} \boldsymbol{S}\|\right\}=\boldsymbol{e}_{\mathrm{obs} .} \cdot\left\{\frac{\delta \mathrm{d} \boldsymbol{S}}{\|\mathrm{d} \boldsymbol{S}\|}-\left(\boldsymbol{n} \cdot \frac{\delta \mathrm{d} \boldsymbol{S}}{\|\mathrm{d} \boldsymbol{S}\|}\right) \boldsymbol{n}\right\} \\
= & \frac{\left[r_{\theta}(\sin i \sin \theta \cos \phi+\cos i \cos \theta)+r(\sin i \cos \theta \cos \phi-\cos i \sin \theta)\right] \cdot\left[r_{\theta} \xi_{r}-r \partial_{\theta} \xi_{r}+r \xi_{\theta}+r_{\theta} \partial_{\theta} \xi_{\theta}\right]}{\left[r^{2}+r_{\theta}^{2}\right]^{3 / 2}} \\
& -\frac{\sin i \sin \phi}{\sin \theta} \frac{\left[-r \partial_{\phi} \xi_{r}+r_{\theta} \partial_{\phi} \xi_{\theta}+\left(r \sin \theta-r_{\theta} \cos \theta\right) \xi_{\phi}\right]}{r\left[r^{2}+r_{\theta}^{2}\right]^{1 / 2}},
\end{aligned}
$$

where we have used the relation $\delta\|\mathrm{d} \boldsymbol{S}\|=\mathrm{d} \boldsymbol{S} \cdot \delta \mathrm{d} \boldsymbol{S} /\|\mathrm{d} \boldsymbol{S}\|=\boldsymbol{n} \cdot \delta \mathrm{d} \boldsymbol{S}$. In the spherical limit, the above expressions become

$$
\begin{aligned}
\mathrm{d} \boldsymbol{S} & =\left(r^{2} \sin \theta \boldsymbol{e}_{r}\right) \mathrm{d} \theta \mathrm{d} \phi \\
\delta(\mathrm{d} \boldsymbol{S}) & =\left\{\left(2 r \sin \theta \xi_{r}+r \cos \theta \xi_{\theta}+r \sin \theta \partial_{\theta} \xi_{\theta}+r \partial_{\phi} \xi_{\phi}\right) \boldsymbol{e}_{r}+\left(-r \sin \theta \partial_{\theta} \xi_{r}+r \sin \theta \xi_{\theta}\right) \boldsymbol{e}_{\theta}+\left(-r \partial_{\phi} \xi_{r}+r \sin \theta \xi_{\phi}\right) \boldsymbol{e}_{\phi}\right\} \mathrm{d} \theta \mathrm{d} \phi, \\
\mu & =\sin i \sin \theta \cos \phi+\cos i \cos \theta \\
\delta \mu & =\frac{[\sin i \cos \theta \cos \phi-\cos i \sin \theta]\left[-\partial_{\theta} \xi_{r}+\xi_{\theta}\right]}{r}-\frac{\sin i \sin \phi}{\sin \theta} \frac{\left(-\partial_{\phi} \xi_{r}+\sin \theta \xi_{\phi}\right)}{r},
\end{aligned}
$$

in full agreement with the expressions previously obtained by Heynderickx et al. (1994) for a non-rotating star with $i=0$, provided one uses the relations:

$\xi_{r}=\delta r, \quad \xi_{\theta}=r \delta \theta, \quad \xi_{\phi}=r \sin \theta \delta \phi$

\subsection{Lagrangian perturbation to the effective gravity}

Although the pulsation equations are given for a cylindrical rotation profile, we will relax this assumption in this section and allow for a general 2D rotation profile when establishing an expression for the Lagrangian perturbation to the effective gravity. Before giving the Lagrangian perturbation to the effective gravity, it is useful to recall various expressions for the unperturbed surface effective gravity:

$\boldsymbol{g}_{\mathrm{eff}}=-\boldsymbol{\nabla} \Psi_{0}+s \Omega^{2} \boldsymbol{e}_{s}=\frac{\boldsymbol{\nabla} P_{0}}{\rho_{0}}=\frac{\partial_{\zeta} P_{0}}{\rho_{0}} \boldsymbol{E}^{\zeta}=-g_{\mathrm{eff}} \boldsymbol{n}$.

The minus sign in the last equation comes from the fact that gravity is pointed inward, $g_{\text {eff }}$ being the norm of $\boldsymbol{g}_{\mathrm{eff}}$. Furthermore, contrarily to what happens in the stellar interior, the $\theta$ derivative of $P_{0}$ vanishes at the surface since the surface is in pressure equilibrium. Any of the above expressions may be used to evaluate the surface effective gravity, although $-\nabla \Psi_{0}+s \Omega^{2} \boldsymbol{e}_{s}$ provides the most accurate numerical results. It is also worth noting that the above expression neglects any contributions from meridional circulation and viscous forces to the equilibrium model (e.g. Rieutord \& Espinosa Lara 2009).

The relative Lagrangian perturbation to the effective gravity is then given by:

$\frac{\delta g_{\mathrm{eff}}}{g_{\mathrm{eff}}}=\frac{\delta\left\|\boldsymbol{g}_{\mathrm{eff}}\right\|}{\left\|\boldsymbol{g}_{\mathrm{eff}}\right\|}=-\frac{\boldsymbol{n} \cdot \delta \boldsymbol{g}_{\mathrm{eff}}}{g_{\mathrm{eff}}}$,

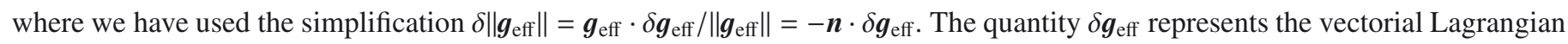
perturbation to the effective gravity. It includes the Lagrangian perturbation to the gradient of the gravitational potential, and the acceleration of a particle tied to the surface, resulting from the oscillatory motions:

$\delta \boldsymbol{g}_{\mathrm{eff}}=-\boldsymbol{\nabla} \Psi-\boldsymbol{\xi} \cdot \boldsymbol{\nabla}\left(\boldsymbol{\nabla} \Psi_{0}\right)+(\omega+m \Omega)^{2} \boldsymbol{\xi}-2 i(\omega+m \Omega) \boldsymbol{\Omega} \times \boldsymbol{\xi}-\boldsymbol{\Omega} \times(\boldsymbol{\Omega} \times \boldsymbol{\xi})$.

Rather than working with the above expression, it is more useful to introduce the equilibrium effective gravity by adding and subtracting $\boldsymbol{\xi} \cdot \boldsymbol{\nabla}\left(s \Omega^{2} \boldsymbol{e}_{s}\right)$ :

$\delta \boldsymbol{g}_{\mathrm{eff}}=-\boldsymbol{\nabla} \Psi+\boldsymbol{\xi} \cdot \boldsymbol{\nabla} \boldsymbol{g}_{\mathrm{eff}}+(\omega+m \Omega)^{2} \boldsymbol{\xi}-2 i(\omega+m \Omega) \boldsymbol{\Omega} \times \boldsymbol{\xi}-\boldsymbol{\Omega} \times(\boldsymbol{\Omega} \times \boldsymbol{\xi})-\boldsymbol{\xi} \cdot \boldsymbol{\nabla}\left(s \Omega^{2} \boldsymbol{e}_{s}\right)$. 

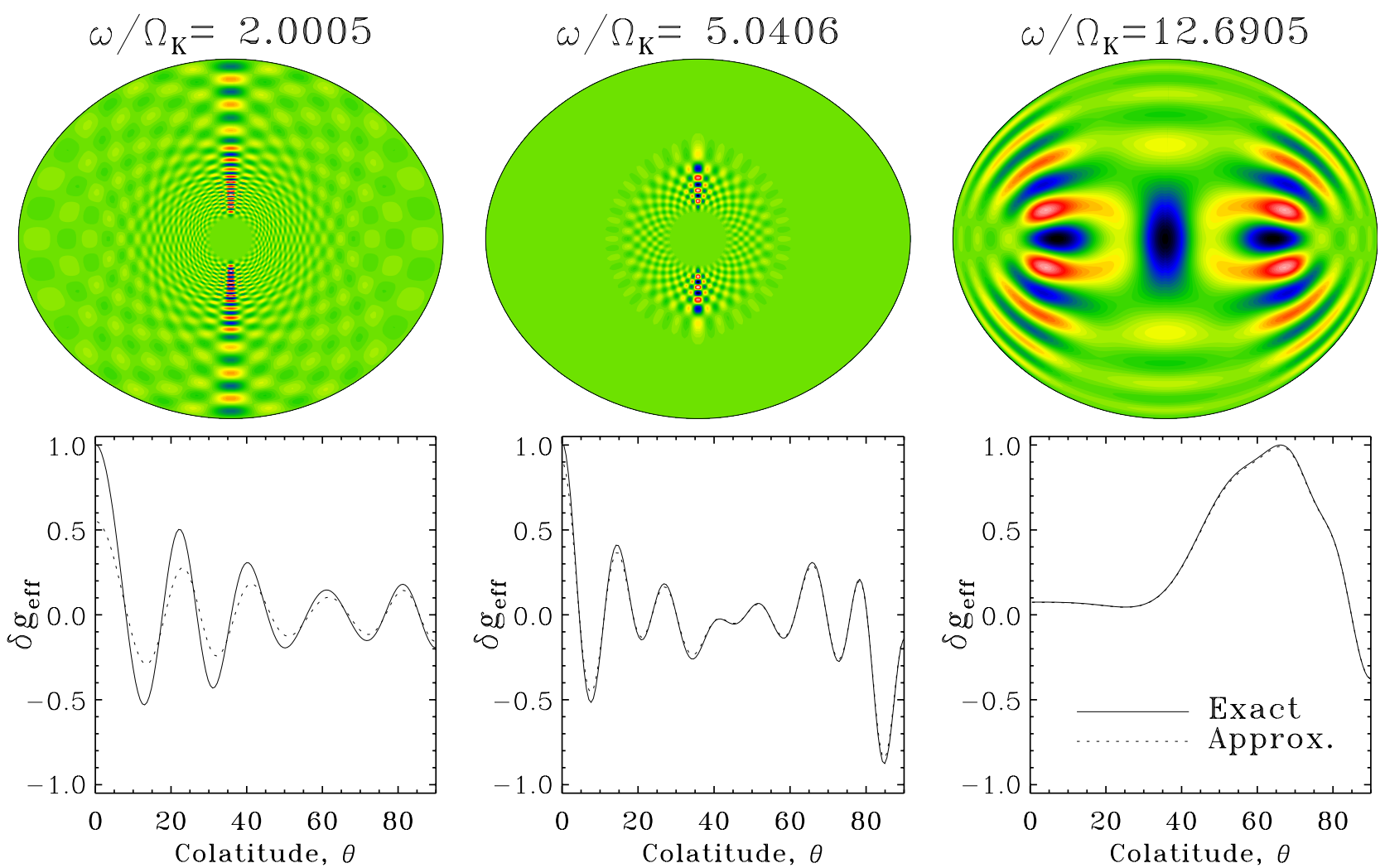

Fig. 2. Comparison between $\delta g_{\text {eff }}$ and $\delta g_{\text {eff }}^{\text {appox. }}$ for three modes. The upper row shows the meridional cross-section of the three modes, whereas the lower row compares the two calculations for the perturbation to the effective gravity at the stellar surface, between one of the poles $\left(\theta=0^{\circ}\right)$ and the equator $\left(\theta=90^{\circ}\right)$. For the middle and right mode, the two curves nearly overlap, making it hard to distinguish them.

After some simplifications based on Poisson's equation and a lengthy derivation described in Appendix B, one obtains the following explicit expression for a general rotation profile:

$$
\begin{aligned}
\delta g_{\mathrm{eff}}= & \frac{\left(r^{2}+r_{\theta}^{2}\right)^{1 / 2} \partial_{\zeta} \Psi}{r r_{\zeta}}-\frac{r_{\theta} \partial_{\theta} \Psi}{r\left(r^{2}+r_{\theta}^{2}\right)^{1 / 2}}+\frac{r \xi_{r}-r_{\theta} \xi_{\theta}}{\left(r^{2}+r_{\theta}^{2}\right)^{1 / 2}}\left\{4 \pi G \rho_{0}-2 \Omega^{2}-\left[\frac{\sin \theta\left(r \sin \theta-r_{\theta} \cos \theta\right)}{r_{\zeta}} \partial_{\zeta}\left(\Omega^{2}\right)+\sin \theta \cos \theta \partial_{\theta}\left(\Omega^{2}\right)\right]\right\} \\
& +\xi_{r}\left\{\frac{r_{\theta} \partial_{\theta} g_{\mathrm{eff}}}{r^{2}+r_{\theta}^{2}}-\frac{\left(2 r-r_{\theta} \cot \theta\right)\left(r^{2}+r_{\theta}^{2}\right)+r r_{\theta}^{2}-r^{2} r_{\theta \theta}}{\left(r^{2}+r_{\theta}^{2}\right)^{2}} g_{\mathrm{eff}}\right\} \xi_{\theta}\left\{\frac{r \partial_{\theta} g_{\mathrm{eff}}}{r^{2}+r_{\theta}^{2}}-\frac{r r_{\theta}\left(-2 r^{2}-3 r_{\theta}^{2}+r r_{\theta \theta}\right)+\left(r^{2}+r_{\theta}^{2}\right) r_{\theta}^{2} \cot \theta}{r\left(r^{2}+r_{\theta}^{2}\right)^{2}} g_{\mathrm{eff}}\right\} \\
& -(\omega+m \Omega)^{2} \frac{r \xi_{r}-r_{\theta} \xi_{\theta}}{\left(r^{2}+r_{\theta}^{2}\right)^{1 / 2}}+\left\{-2 i(\omega+m \Omega) \Omega \xi_{\phi}+\left[\frac{r \xi_{r}-r_{\theta} \xi_{\theta}}{r_{\zeta}} \sin \theta \partial_{\zeta}\left(\Omega^{2}\right)+\xi_{\theta} \sin \theta \partial_{\theta}\left(\Omega^{2}\right)\right]\right\} \frac{r \sin \theta-r_{\theta} \cos \theta}{\left(r^{2}+r_{\theta}^{2}\right)^{1 / 2}} .
\end{aligned}
$$

It is worth noting that in the above expression, all of the terms involving a $\zeta$ derivative of one of the perturbed quantities are divided by $r_{\zeta}$. The remaining terms involve no $\zeta$ derivatives whatsoever. This characteristic is what one would expect for a quantity which is independent of the mapping.

If a cylindrical rotation profile is used instead, the terms in square brackets simplify to the following expressions, respectively:

$$
s \partial_{s}\left(\Omega^{2}\right), \quad\left(\sin \theta \xi_{r}+\cos \theta \xi_{\theta}\right) s \partial_{s}\left(\Omega^{2}\right)
$$

In the non-rotating limit, Eq. (35) reduces to:

$\delta g_{\mathrm{eff}}=\partial_{r} \Psi+4 \pi G \rho_{0} \xi_{r}-\frac{2 \xi_{r}}{r} g_{\mathrm{eff}}-\omega^{2} \xi_{r}$

in full agreement with Dupret et al. (2002).

Given the complexity of Eq. (35), it is interesting to see if it can be approximated by a simpler expression. We consider the following approximation:

$\delta g_{\mathrm{eff}}^{\text {approx. }}=-(\omega+m \Omega)^{2} \boldsymbol{\xi} \cdot \boldsymbol{n}=-(\omega+m \Omega)^{2} \frac{r \xi_{r}-r_{\theta} \xi_{\theta}}{\left(r^{2}+r_{\theta}^{2}\right)^{1 / 2}}$.

Figure 2 compares $\delta g_{\mathrm{eff}}$ and $\delta g_{\mathrm{eff}}^{\text {approx. }}$ for three different modes - two gravito-inertial modes and one p-mode. As can be seen in this figure, $\delta g_{\text {eff }}^{\text {approx. }}$ is a very good approximation for $\mathrm{p}$ - and g-modes with a sufficiently high frequency. It is only for low frequency g-modes that the difference between the two expressions becomes non-negligible. 
D. R. Reese et al.: Mode visibilities in rapidly rotating stars
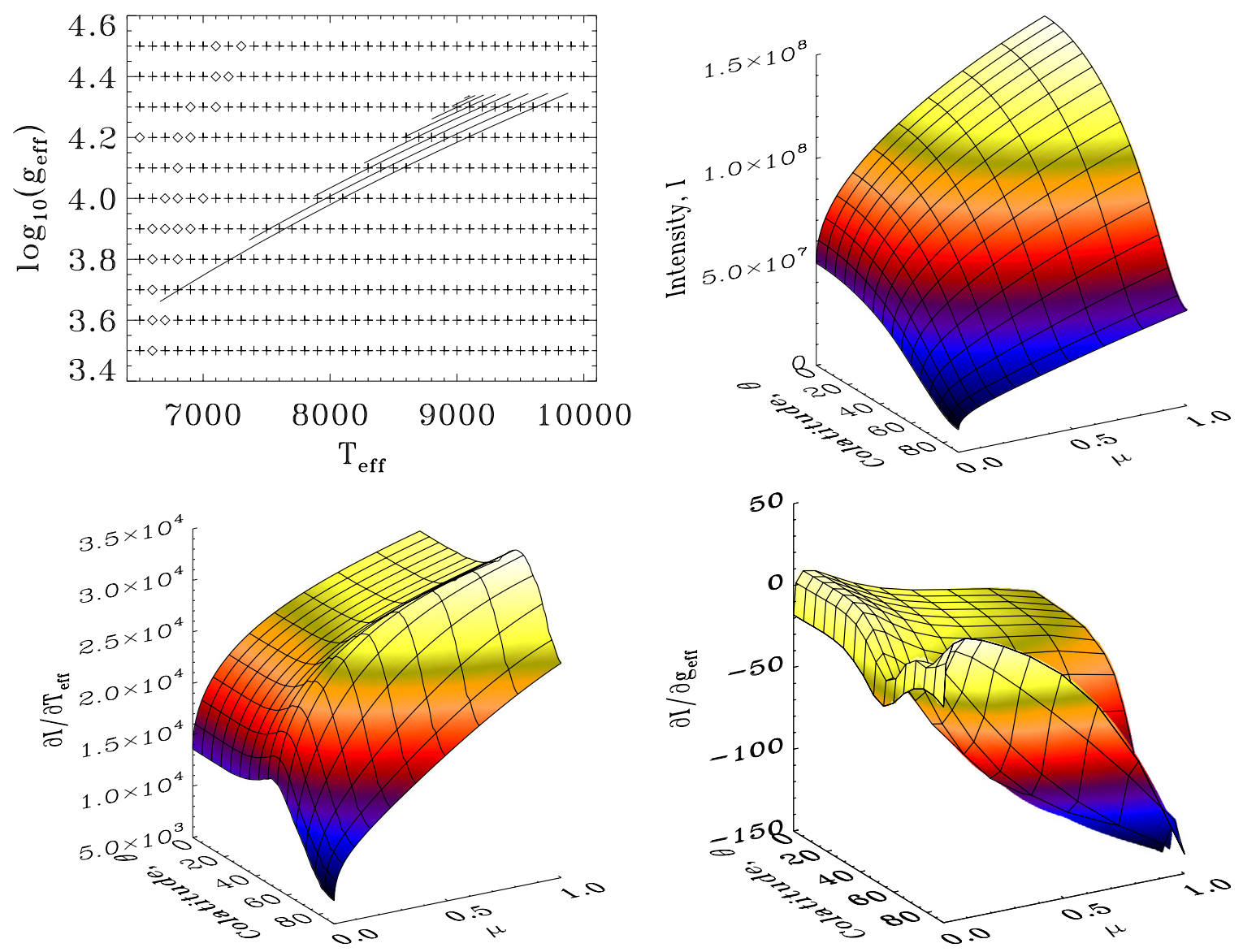

Fig. 3. (Upper left panel) Effective temperatures and gravities of the Kurucz model atmospheres (symbols) and the $2 M_{\odot}$ models (continuous lines; the lower longer lines correspond to more rapid rotation). The pluses (+) represent initial Kurucz atmospheres whereas the diamonds $(\diamond)$ represent missing points which were interpolated from the neighbouring atmosphere models. (Remaining panels) Specific intensities and its partial derivatives for a $2 M_{\odot}, 0.8 \Omega_{\mathrm{c}}$ model, as a function of colatitude and $\mu$.

\subsection{Lagrangian perturbation to the temperature}

We then turn our attention to the relative Lagrangian perturbations of the effective temperature, $\delta T_{\text {eff }} / T_{\text {eff }}$. Given that the pulsation calculations are done in the adiabatic approximation, this quantity is not readily available. We will therefore content ourselves with the approximation $\delta T_{\text {eff }} / T_{\text {eff }} \approx \delta T / T_{0}$. We note that this approximation is different from the approximation $\delta T_{\text {eff }} \approx \delta T$ given that $T_{\text {eff }}$ is latitude-dependant whereas $T_{0}$ is not in barotropic stellar models. As was pointed out in Sect. 2.5, a modified version of the mechanical boundary condition was needed in order to obtain a non-zero value for $\delta T$ at the surface. One may then wonder if other approximations may be more suitable, such as applying the boundary condition $\delta p=0$ high up in the stellar atmosphere and extracting $\delta T$ at a deeper optical depth, or working with the Eulerian temperature fluctuation instead. The main difficulty with the first option is that the stellar models used in the present study do not include an atmosphere ${ }^{2}$. Hence, it is not clear at what depth (which is likely to be latitude-dependant), one should extract the Lagrangian temperature variations. Using the Eulerian temperature variations also has problems of its own. Indeed, at the surface, the Eulerian temperature variations are dominated by the the advection term, thereby making them an order of magnitude larger than the Lagrangian variations, and with the opposite sign for the most part. Such variations do not seem the most appropriate from a physical point of view, since the intensity variations are more likely to result from local temperature variations physically present in the fluid, i.e. Lagrangian variations. Hence, given the limitations of our models and pulsation calculations, using the Lagrangian temperature variations along with the modified mechanical boundary condition seems like the best choice. Nonetheless, according to Dupret et al. (2002) and Dupret et al. (2003), adiabatic calculations of $\delta T / T_{0}$ are not reliable in the superficial layers and consequently lead to a poor approximation of $\delta T_{\text {eff }} / T_{\text {eff }}$. In order to obtain $\delta T_{\text {eff }} / T_{\text {eff }}$ accurately, one would need to do a full non-adiabatic calculations including a detailed treatment of the atmosphere such as what is done in Dupret et al. (2002), using 2D rotating models in thermal equilibrium, such as what is being developed in the ESTER project (Rieutord \& Espinosa Lara 2009; Espinosa Lara 2010).

The quantity $\delta T / T_{0}$ can be deduced very simply from $\delta p / P_{0}$ via the adiabatic relation:

$\frac{\delta T}{T_{0}}=\frac{\Gamma_{2}-1}{\Gamma_{2}} \frac{\delta p}{P_{0}}$

where $\Gamma_{2}$ is the second adiabatic exponent, given by the expression $\frac{\Gamma_{2}-1}{\Gamma_{2}}=\left(\frac{\partial \ln T}{\partial \ln p}\right)_{\mathrm{ad}}$.

\footnotetext{
2 The Kurucz atmospheres described in the following section are only used to calculate the emergent intensities as a function of $T_{\mathrm{eff}}$ and $g_{\mathrm{eff}}$, and are not "joined" to the present models.
} 


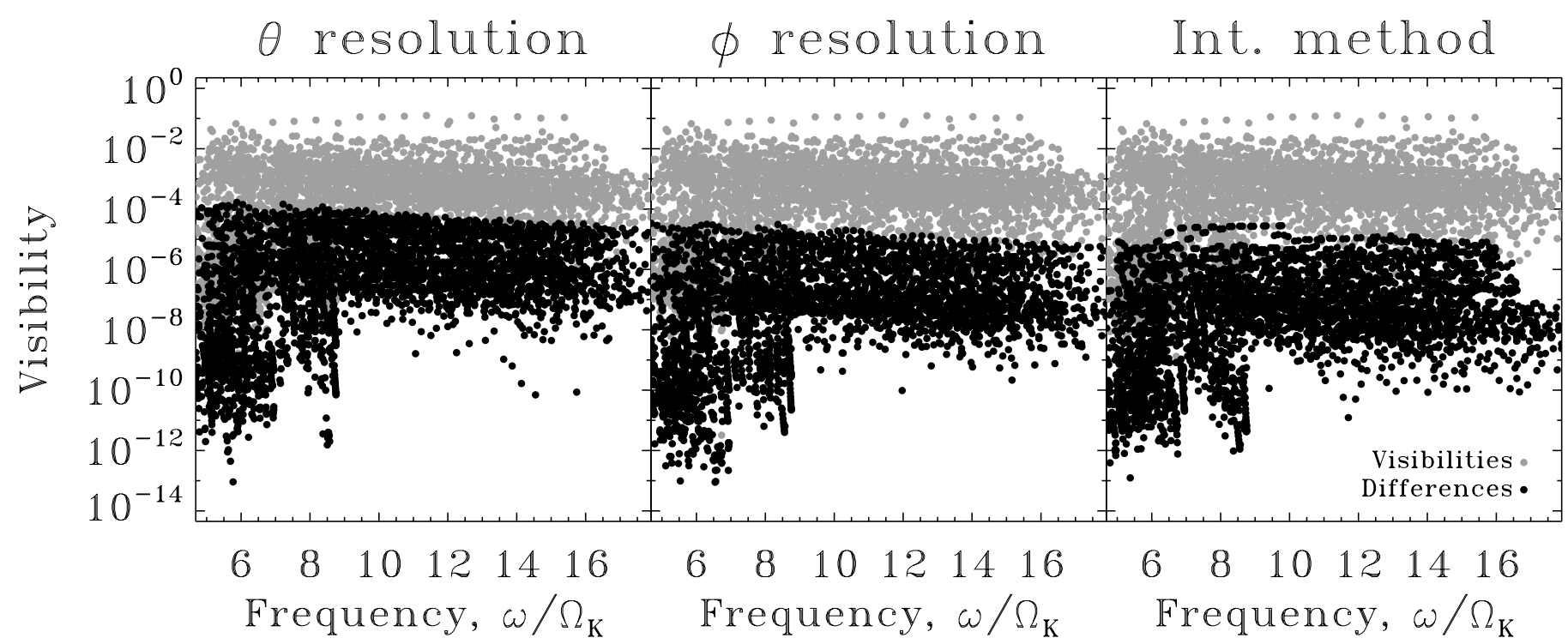

Fig. 4. Visibility calculations and differences for a set of 3197 modes, obtained using two different $\theta$ resolutions, $N_{\theta}=300$ and 2000 (left panel), two different $\phi$ resolutions, $N_{\phi}=720$ and 4000 (middle panel), and two different integration methods in the theta direction (right panel). These modes were calculated in a $2.0 M_{\odot}$ model at $0.6 \Omega_{\mathrm{C}}$, with an inclination angle $i=10^{\circ}$. If $i=80^{\circ}$ is used instead, the differences are reduced.

\subsection{Specific intensities}

Contrarily to the non-rotating case, the effective temperature and gravity of the equilibrium model depend on the latitude. Consequently, it is necessary to find the appropriate set of specific intensities and their derivatives for each latitude. We therefore calculated a grid of Kurucz atmospheres with solar composition which spans the relevant temperature and gravity ranges. We used the ATLAS $9 \operatorname{code}^{3}$ (Kurucz 1993) in a modified form so as to include the convective prescription of Canuto et al. (1996), known as CGM (for more details see Heiter et al. 2002; and Barban et al. 2003). Due to convergence problems, some of the grid points were missing and had to be interpolated from neighbouring points. The final grid is illustrated in Fig. 3 (upper left panel) as is $\log _{10}\left(g_{\text {eff }}\right)$ as a function of $T_{\text {eff }}$ for a set of $2 M_{\odot}$ models with rotation rates ranging from $0 \%$ to $80 \%$ of the critical break-up rotation rate.

For each grid point, we then approximated the $\mu$ dependence through a Claret type law (Claret 2000) using a least-squares fit. This yielded a set of five coefficients $\left(I(\mu=1), I(1) a_{1}, I(1) a_{2}, I(1) a_{3}, I(1) a_{4}\right)$ from which it is possible to calculate $I$ and $\partial I / \partial \mu$ for any $\mu$. Using this approach rather than dealing directly with the original values for $I$ yields better numerical results, both for interpolating $I$ and especially for taking its $\mu$ derivative.

We then successively applied cubic spline interpolations, first as a function of $\log _{10}\left(g_{\text {eff }}\right)$, then as a function of $T_{\text {eff }}$. This yielded b-spline coefficients from which it is possible to deduce the Claret coefficients and their derivatives for any value of $\log _{10}\left(g_{\mathrm{eff}}\right)$ and $T_{\text {eff }}$ within the relevant range. From this representation of $I$, we then calculated the Claret coefficients for $I, \partial I / \partial g_{\text {eff }}$ and $\partial I / \partial T_{\text {eff }}$ as a function of latitude for each stellar model. The remaining three panels of Fig. 3 display these quantities as a function of $\mu$ and colatitude, $\theta$, for a $2 M_{\odot}$ model at $80 \%$ of the critical rotation rate. As is illustrated in these panels, both limb and gravity darkening are taken into account. The latitude-dependant Claret coefficients were then projected onto the spherical harmonic basis, truncated at 40 terms. This last step allows us to easily interpolate these coefficients onto a denser latitudinal grid during the visibility calculations.

\subsection{Visibility integrals}

Once the above quantities are calculated over the stellar surface, the visibilities may be evaluated numerically. However, rather than using the time dependant form given in Eq. (18), it is more useful to extract the amplitude and phase of $\Delta E(t)$. To do so, we cast Eq. (18) into the following schematic form:

$$
\begin{aligned}
\Delta E(t) & =\mathfrak{R}\left\{\iint_{\text {Vis.Surf. }}[A(\theta, \phi)+i B(\theta, \phi)] \mathrm{e}^{\mathrm{i} m \phi+i \omega t} \mathrm{~d} \theta \mathrm{d} \phi\right\} \\
& =\mathfrak{R}\left\{\iint_{\text {Vis.Surf. }}[(A \cos (m \phi)-B \sin (m \phi))+i(A \sin (m \phi)+B \cos (m \phi))] \mathrm{e}^{\mathrm{i} \omega t} \mathrm{~d} \theta \mathrm{d} \phi\right\} \\
& =\cos (\omega t) \underbrace{\iint_{\text {Vis.Surf. }}(A \cos (m \phi)-B \sin (m \phi)) \mathrm{d} \theta \mathrm{d} \phi}_{\mathcal{A}}-\sin (\omega t) \underbrace{\iint_{\text {Vis.Surf. }}(A \sin (m \phi)+B \cos (m \phi)) \mathrm{d} \theta \mathrm{d} \phi}_{\mathcal{B}}
\end{aligned}
$$

where $A(\theta, \phi)$ and $B(\theta, \phi)$ are real. This leads to:

$\Delta E(t)=C \cos (\omega t+\varphi)$

\footnotetext{
3 See http://kurucz.harvard.edu
} 
where $C=\sqrt{\mathcal{A}^{2}+\mathcal{B}^{2}}$ is the amplitude and $\varphi=\arctan (\mathcal{B} / \mathcal{A})$ the phase. Based on symmetry considerations with respect to the variable $\phi$, the term $\mathcal{B}$ cancels out in the adiabatic case, thereby leading to $C=|\mathcal{A}|$ and $\varphi=0$ or $\pi$. If, however, one tries to simulate non-adiabatic effects by introducing a phase-lag in the temperature variations, then the term $\mathcal{B}$ no longer cancels out.

The integrals $\mathcal{A}$ and $\mathcal{B}$ are evaluated numerically. The surface is discretised in the $\theta$ direction, using a Gauss-Legendre collocation grid with typically $N_{\theta}=300$ points, and in the $\phi$ direction using a uniform grid with a typical resolution of $N_{\phi}=720$. The integration weights in the $\theta$ direction are deduced from a Gauss quadrature, whereas those in the $\phi$ direction are uniform. Figure 4, shows the differences which result from increasing either $N_{\theta}$ (left panel) or $N_{\phi}$ (middle panel) in a $2 M_{\odot}$ stellar model, rotating at $60 \%$ of the critical rotation rate. As can be seen, these differences are several orders of magnitude smaller than the visibilities themselves.

At each point on the surface, the following condition is evaluated to determine whether or not the surface element is facing the observer and should be included in the integral:

$\mu \geq 0$

where $\mu$ is given in Eq. (24). We note that this condition is only valid if no obstacles are present between the surface element and the observer, such as what could happen, for instance, in the more distorted SCF models with concavities at the poles (due to highly differential rotation) and an orientation which is not too far from equator-on. For such configurations, a more general condition based on a z-buffer or ray-tracing approach would be required.

One possible concern is that using a Gauss quadrature implicitly assumes a spectral decomposition of the function which is being integrated, which could lead to a Gibbs phenomena near the cutoff between the visible and hidden side of the star. However, given that the Gibbs phenomena is oscillatory, one can expect its integrated contribution do be small. Nonetheless, we carried out a test on the same model, using both Gauss quadrature and a trapezoidal integration. As can be seen in the right panel of Fig. 4, the two approaches yielded very similar results, showing that the Gibbs phenomena does not play an important role.

\subsection{Comparison with $1 D$ case for non-rotating stars}

It is also important to check that in the non-rotating case, visibility calculations based on the above 2D integrations agree with the results obtained with a simpler 1D integration, as is commonly used in other works (e.g. Heynderickx et al. 1994). In order to obtain the 1D integrals, one starts with a given mode, which in this case will be proportional to a particular spherical harmonic, $Y_{\ell}^{m}(\theta, \phi)$, re-expresses this spherical harmonic in terms of a new coordinate system with the $z^{\prime}$-axis aligned with the line of sight via the following decomposition (Edmonds 1960):

$Y_{\ell}^{m}(\theta, \phi)=\sum_{m^{\prime}=-\ell}^{\ell} d_{m m^{\prime}}^{\ell}(i) Y_{\ell}^{m^{\prime}}\left(\theta^{\prime}, \phi^{\prime}\right)$

and calculates the integrated contribution from each of the $Y_{\ell}^{m^{\prime}}\left(\theta^{\prime}, \phi^{\prime}\right)$. In this new coordinate system, the visible surface corresponds to a hemisphere. Consequently, all of the non-axisymmetric terms $m^{\prime} \neq 0$ cancel out and only the term $d_{m 0}^{\ell}(i) Y_{\ell}^{0}\left(\theta^{\prime}, \phi^{\prime}\right)$ remains. This leads to the following expression:

$\Delta E(t)=\frac{r^{2} d_{m 0}^{\ell}(i)}{d^{2}} \mathfrak{R}\left\{\left[\frac{\delta \hat{T}_{\mathrm{eff}}}{T_{\mathrm{eff}}} \int_{0}^{1} \frac{\partial I}{\partial \ln T_{\mathrm{eff}}} Y_{0}^{\ell} \mu^{\prime} \mathrm{d} \mu^{\prime}+\frac{\delta \hat{g}_{\mathrm{eff}}}{g_{\mathrm{eff}}} \int_{0}^{1} \frac{\partial I}{\partial \ln g_{\mathrm{eff}}} Y_{0}^{\ell} \mu^{\prime} \mathrm{d} \mu^{\prime}+\frac{\hat{\xi}_{r}}{r}(2+\ell)(1-\ell) \int_{0}^{1} I Y_{0}^{\ell} \mu^{\prime} \mathrm{d} \mu^{\prime}\right] \mathrm{e}^{\mathrm{i} \omega t}\right\}$

where

$d_{m 0}^{\ell}(i)=\varepsilon \sqrt{\frac{4 \pi}{2 \ell+1}} Y_{\ell}^{m}(\theta=i, \phi=0), \quad \mu^{\prime}=\cos \theta^{\prime}, \xi_{r}=\hat{\xi}_{r} Y_{\ell}^{m}, \quad \delta T_{\mathrm{eff}}=\left(\delta \hat{T}_{\mathrm{eff}}\right) Y_{\ell}^{m}, \quad$ and $\quad \delta g_{\mathrm{eff}}=\left(\delta \hat{g}_{\mathrm{eff}}\right) Y_{\ell}^{m}$,

and $\varepsilon= \pm 1$ depending on the sign convention used in defining the spherical harmonics, and the values of $\ell$ and $m$. Figure 5 shows the differences between the two approaches. As can be seen, the results are very similar, thereby validating the $2 \mathrm{D}$ integrations.

\subsection{Comparison with Lignières et al. (2006) and Lignières \& Georgeot (2009)}

Before going on to describe the results, we carry out one last test in which we compare the 2D integration method described here with the harmonic projection method used in Lignières et al. (2006) and Lignières \& Georgeot (2009). In order to carry out the comparison, we calculate simplified disk-integration factors, $D(i)$, by only integrating the temperature fluctuations over the visible surface. We avoid using the intensity fluctuations deduced from the Kurucz atmospheres, as this would introduce the effects of limb darkening and a $\phi$ dependence of a different form than $\mathrm{e}^{\mathrm{i} m \phi}$, which is not currently implemented in the method by Lignières \& Georgeot (2009). Hence, the disk-integration factors are given by:

$D(i) \cos (\omega t+\psi)=\mathfrak{R}\left\{\frac{1}{\pi R_{\text {eq }}^{2}\langle\delta T\rangle} \iint_{\text {Vis.Surf. }} \delta T \boldsymbol{e}_{\mathrm{obs}} \cdot \mathrm{d} \boldsymbol{S}\right\}$, where $\langle\delta T\rangle=\left(\frac{1}{S} \iint_{\mathrm{S}}|\delta T|^{2} \mathrm{~d} S\right)^{1 / 2}$,

and where $S$ is the total surface and $\psi$ a suitably chosen phase. The disk-integration factors are normalised in the same way as is done in Lignières et al. (2006) and Lignières \& Georgeot (2009), which leads to $D(0)=1$ if $\delta T \equiv 1$. Figure 6 shows the differences between the two approaches for a model rotating at $0.8 \Omega_{\mathrm{C}}$. As can be seen, the differences are quite small relative to the visibilities themselves. At this point, it is helpful to remember that the curve which delimits the visible surface, is approximated 


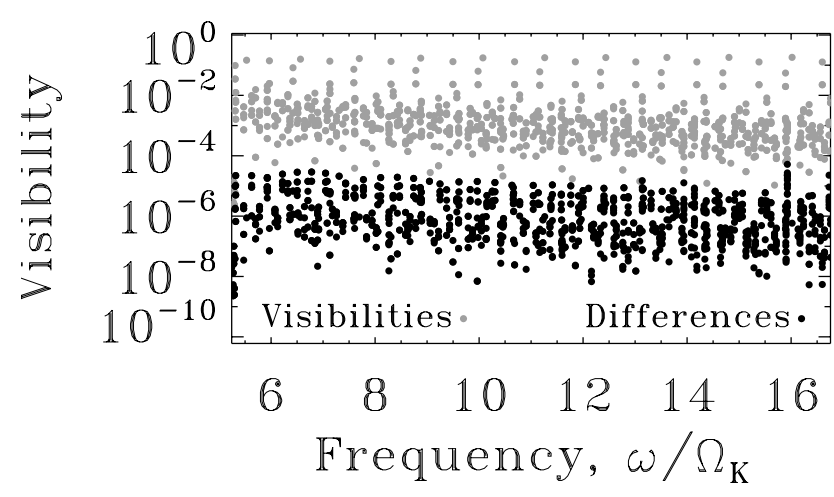

Fig. 5. Visibility calculations and differences for a set of 1223 modes in a non-rotating model, using $1 \mathrm{D}$ and 2D integrations. The latitudinal resolution is $N_{\theta}=300$ for both approaches, and $N_{\phi}=720$ points are used in the azimuthal direction for the 2D integrations. The inclination angle is $i=10^{\circ}$. Note: because of mode degeneracy, there are much fewer frequencies.

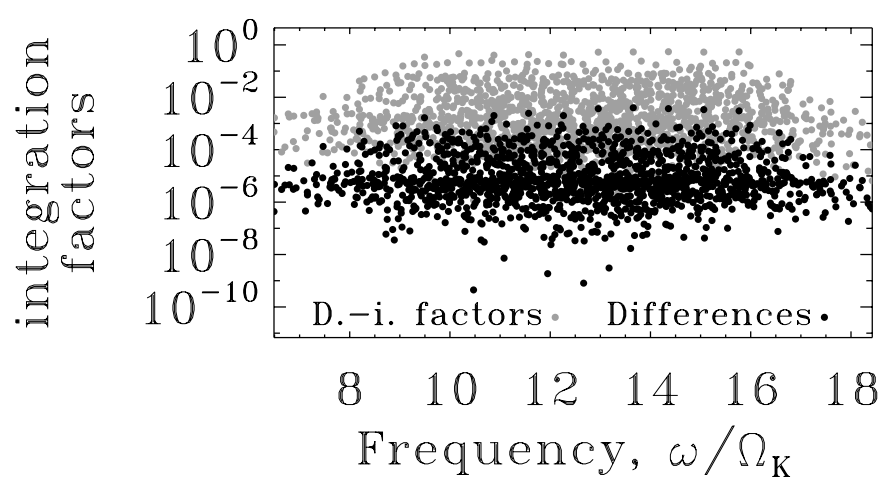

Fig. 6. Simplified disk-integration factors and differences for a set of 1507 modes in a model rotating at $0.8 \Omega_{\mathrm{C}}$, using $2 \mathrm{D}$ integrations and the harmonic projection method described in Lignières et al. (2006) and Lignières \& Georgeot (2009). The resolutions are $N_{\theta}=300$ for both approaches, and $N_{\phi}=720$ for the $2 \mathrm{D}$ integrations. The inclination angle is $i=10^{\circ}$. Note: due to symmetries in the simplified visibility calculations, the visibilities of some modes cancel out and were therefore not included in this plot (see text for details).

as the intersection of a plane and the stellar surface in the harmonic projection method. One can expect such an approximation to become less good at high rotation rates due to the strong centrifugal deformation, but as shown in Fig. 6 the effect remains small, in full agreement with the results presented in Lignières \& Georgeot (2009), based on a Roche model.

Given the simplified form given in Eq. (45), it turns out that modes with $|m| \geq 2$ and such that $(-1)^{m+1} \delta \hat{T}(\pi-\theta)=\delta \hat{T}(\theta)$, i.e. even modes when $m$ is odd and vice versa, have zero disk-integration factors, regardless of orientation. Consequently, these lead to numerical results around $10^{-16}$ or less, for both methods, and were therefore not represented in Fig. 6. Appendix C explains why these disk-integration factors cancel out.

\section{Overall visibilities}

In what follows we will first focus on mode visibilities in the CoRoT photometric before dealing with amplitude ratios between different bands.

\subsection{General results}

Figure 7 shows the visibilities of modes within acoustic frequency ranges for a set of $2 M_{\odot}$ stellar models at 9 different rotation rates (1 for each row). The columns correspond to 4 different inclinations. Given that the intrinsic mode amplitudes cannot currently be predicted, as this would require a full non-linear development, we normalised the modes so that the maximal displacement within the entire star, $\|\xi\|_{\max }$, multiplied by the square of the co-rotating frequency, $(\omega+m \Omega)^{2}$, is constant. This tends to favour acoustic modes, for which the maximal amplitude is near the surface. Multiplying by the square of the frequency yields a near constant value for the visibilities of modes with similar $\ell$ and $m$ in the acoustic asymptotic regime, for the non-rotating model (upper row). The different colours indicate the $\ell$ value which would carry on from $\Omega=0$ through a mode-following procedure (e.g. Lignières et al. 2006). In Lignières \& Georgeot (2008; see also Reese 2008), a different set of quantum numbers $(\tilde{n}, \tilde{\ell}, m)$ is introduced. This set of quantum numbers is better adapted to island modes, the rapidly rotating equivalent of modes with low $\ell-|m|$ values. The number $\tilde{n}$ corresponds the number of nodes along the underlying ray path associated with these modes, whereas $\tilde{\ell}$ is the number of nodes transverse to this ray path. In the last row of Fig. 7 , the visibilities of the $0.8 \Omega_{\mathrm{C}}$ model are repeated, but using a colour scheme which indicates the value of $\tilde{\ell}$.

A first trend which appears at rapid rotation rates is that the equator-on configurations tend to have larger amplitudes. This is simply due to the fact that we normalise the visibilities with respect to the stellar luminosity in the observer's direction. Given that the star is less luminous equator-on, this leads to larger relative amplitudes. If instead we normalise by the pole-on luminosity, such as what is done in Lignières et al. (2006), the amplitudes show a much smaller increase, due to the equatorial focusing of island modes, as will be discussed in the following section.

A second effect which can be seen in these panels is that the pulsation spectra become more and more complex as $\Omega$ increases. This is caused by several factors. First of all, as opposed to the non-rotating case, modes with the same $n$ and $\ell$ values but different $m$ values have different frequencies. At small (uniform) rotation rates, this leads to frequency multiplets which are evenly spaced and which span a small frequency range. As the rotation rate increases, these multiplets become uneven and they start to overlap, thereby leading to a complex spectrum. Secondly, modes with intermediate $\ell-|m|$ values become chaotic modes at rapid rotation rates. This causes their node spacing to become uneven and is likely to make cancellation effects less efficient than in the nonrotating case (Lignières \& Georgeot 2009). Accordingly, the number of modes which are visible above a given threshold increases. One may wonder if this can explain the irregular nature of pulsation spectra in $\delta$ Scuti stars. Up to some extent yes, but not entirely. 
D. R. Reese et al.: Mode visibilities in rapidly rotating stars

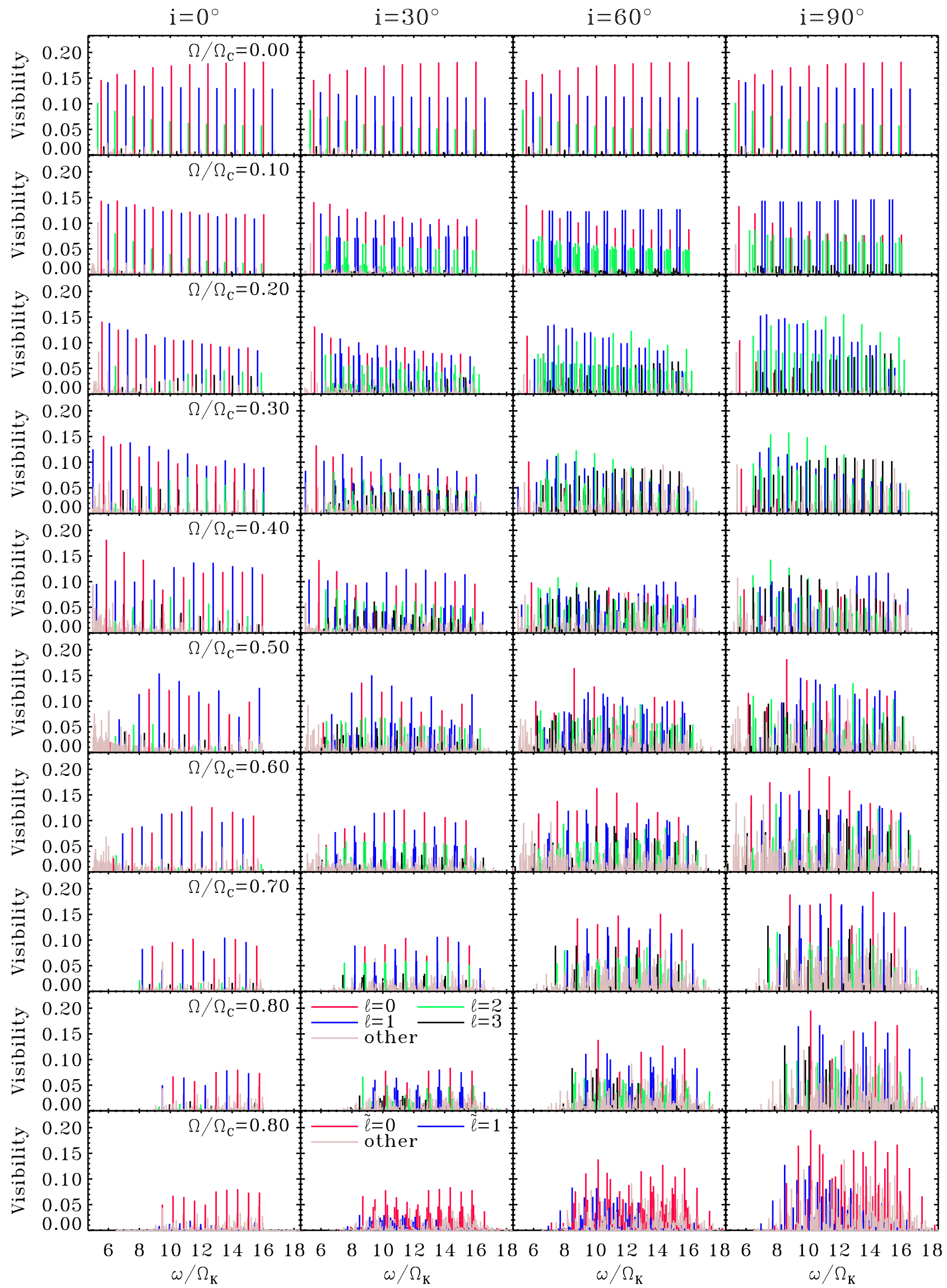

Fig. 7. Mode visibilities for $2 M_{\odot}$ stellar models at 9 different rotation rates, and 4 different inclinations $\left(i=0^{\circ}\right.$ corresponds to a pole-on configuration). The colours correspond to the $\ell$ value carried on from $\Omega=0$, except for the last row where they correspond to $\tilde{\ell}$. (This figure is available in color in electronic form.) 


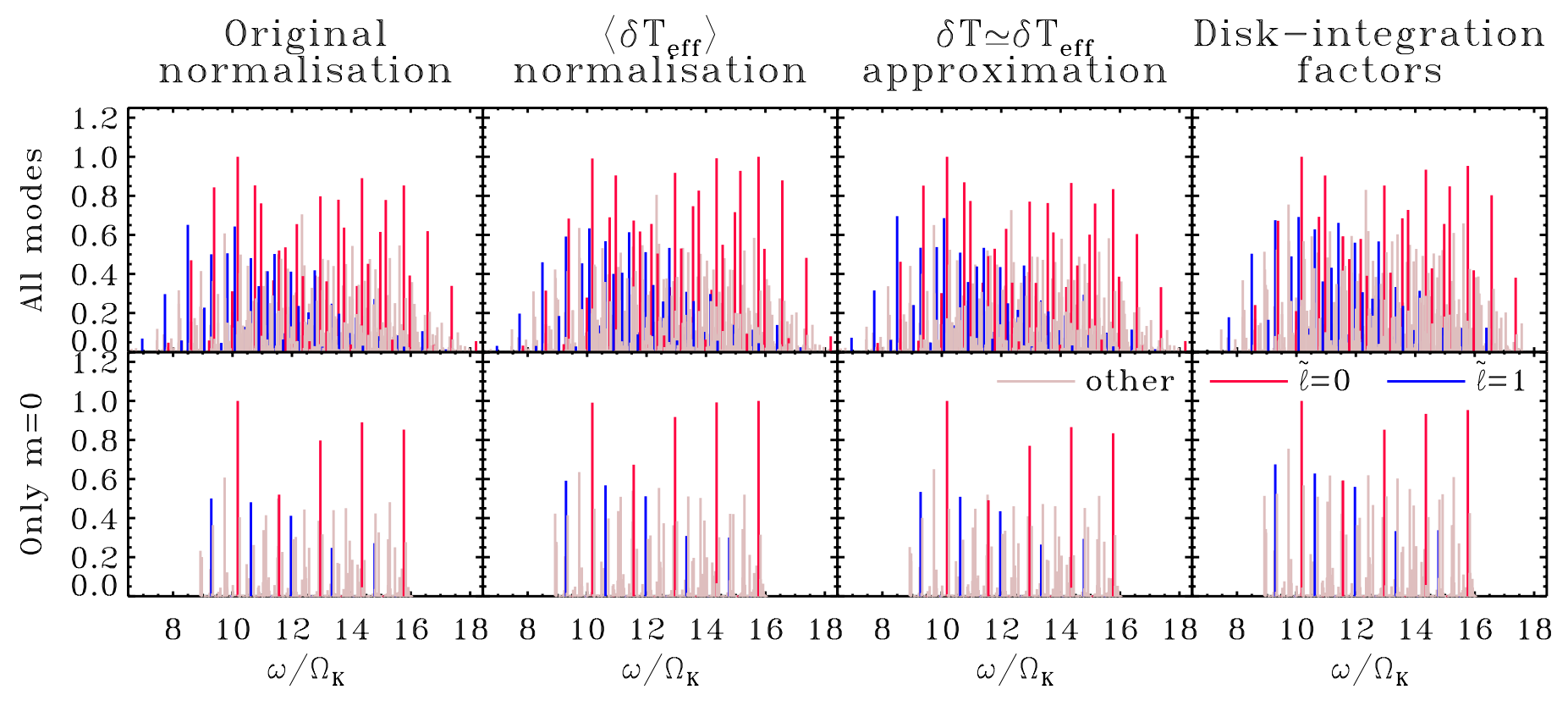

Fig. 8. Mode visibilities calculated or normalised in alternate ways for the model rotating at $\Omega=0.8 \Omega_{\mathrm{C}}$, as viewed from the equator. The upper shows the visibilities of all of the modes whereas the lower panel only includes axisymmetric $(m=0)$ modes. See text for details. (This figure is available in color in electronic form.)

In both cases, the frequencies are distributed so that no simple regularities stand out. However, observations show a somewhat larger dispersion in amplitudes, with only a few high-amplitude modes. This suggests that non-linear effects lead to intrinsic mode amplitudes which cannot be described in a simple way as is done here with the ad hoc normalisation, thus making it difficult to identify modes from visibilities alone.

Another important effect is the relative simplicity of the frequency spectra for the pole-on orientation, and to a lesser extent for $i=30^{\circ}$, even in the most rapidly rotating models. Indeed, non-axisymmetric modes cancel out in the pole-on configuration, and the $\tilde{\ell}=0$ modes, i.e. the rotating counterparts to $\ell=0$ and 1 modes, stand out. This means that such an orientation facilitates interpreting the oscillation spectrum of rapidly rotating stars. Of course, it is also more difficult to identify pole-on stars as rapid rotators, since spectroscopic observations will only reveal narrow absorption lines due to the unknown sin $i$ factor (although the overall shape of the spectrum will differ substantially from that of a black-body at a single temperature). Interferometric studies have, however, been able to confirm that Vega is a rapidly rotating star, seen nearly pole-on $\left(i=4.54^{\circ}\right.$, Peterson et al. 2006). This recently motivated a search for pulsation modes in this star. Böhm et al. (2012) found some stellar variability, but were unable to confirm the presence of stellar oscillations, thereby showing the need for further observations with larger instruments. It is also necessary to search for other pole-on rapid rotators to see if they exhibit stellar pulsations.

In the equator-on configurations, the visibilities of the chaotic modes increases relatively to those of the island modes, thereby making it harder to distinguish the two. This result partially agrees with the results presented in Lignières \& Georgeot (2009), although we do note that here, some of the island modes still tend to remain more visible whereas in Lignières \& Georgeot (2009) the two had comparable amplitudes. To understand where this difference comes from, we tried various manipulations. In Fig. 8, we show alternate ways of calculating or normalising the visibilities for the model rotating at $\Omega=0.8 \Omega_{\mathrm{C}}$. The top row shows the visibilities of all of the modes, whereas the bottom row corresponds to axisymmetric modes $(m=0)$ only. The first column uses the original normalisation, so that the upper left panel of Fig. 8 is identical to the lower right panel of Fig. 7. The second column shows shows what happens if the modes are normalised by $\left\langle\delta T_{\text {eff }}\right\rangle$ rather than $\omega^{2}\|\xi\|_{\max }$. The third column shows what happens if we use the approximation $\delta T \simeq \delta T_{\text {eff }}$ instead of $\delta T / T \simeq \delta T_{\text {eff }} / T_{\text {eff }}$. Finally, the last column shows the disk-integration factors as given in Eq. (45). For the sake of legibility, we divided the visibilities in each panel by the maximal value, so that the results are between 0 and 1. As can be seen, the most visible modes still tend to be island modes regardless of how the visibilities are calculated and whether or not only axisymmetric modes are kept. Hence, another explanation is needed for the difference between the present results and those of Lignières \& Georgeot (2009). One difference between the two studies is the boundary condition on the pressure perturbation. Indeed, in the present study, we set the vertical gradient of $\delta p / P_{0}$ to zero at the surface (Eq. 15), in order to avoid having $\delta T=0$ at the surface. Lignières \& Georgeot (2009) set $\delta p=0$ instead. This however does not lead to $\delta T=0$ in their case, since they are dealing with a polytropic model, where the pulsation equations become singular at the surface. This difference in boundary condition may have an important impact on the temperature fluctuations at the surface which dominate the mode visibilities presented here, although it is hard to test since applying the condition $\delta p=0$ with SCF models would only lead to $\delta T=0$. One possibility would be to apply the condition Eq. (15) to polytropic models.

Finally, Fig. 9 shows the separate contributions coming from the $g_{\text {eff }}, T_{\text {eff }}$ and $\mathrm{d} S$ variations. As can be seen in the figure, the effective temperature fluctuations are the most dominant effect. This is because effective gravity plays a small role in the specific intensity emitted by an atmosphere. The surface deformation plays a small role in acoustic modes because it is proportional to the displacement rather than the displacement times the square of the frequency. 
D. R. Reese et al.: Mode visibilities in rapidly rotating stars

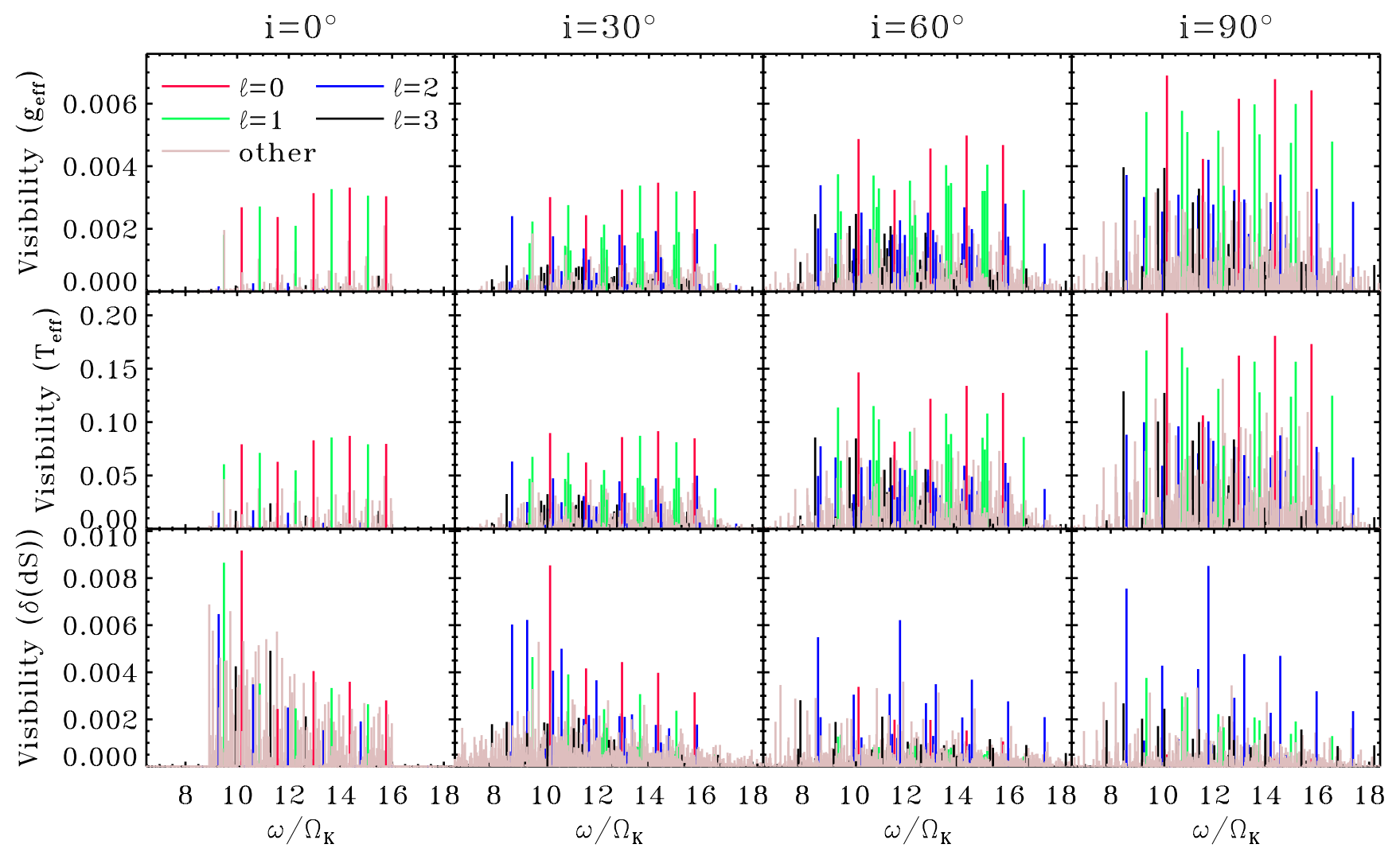

Fig. 9. Separate contributions to the visibilities, coming from the $g_{\text {eff }}, T_{\text {eff }}$ and the $\mathrm{d} S$ variations. These are calculated for the model at $\Omega=0.8 \Omega_{\mathrm{C}}$ (which corresponds to the before last row of Fig. 7). (This figure is available in color in electronic form.)

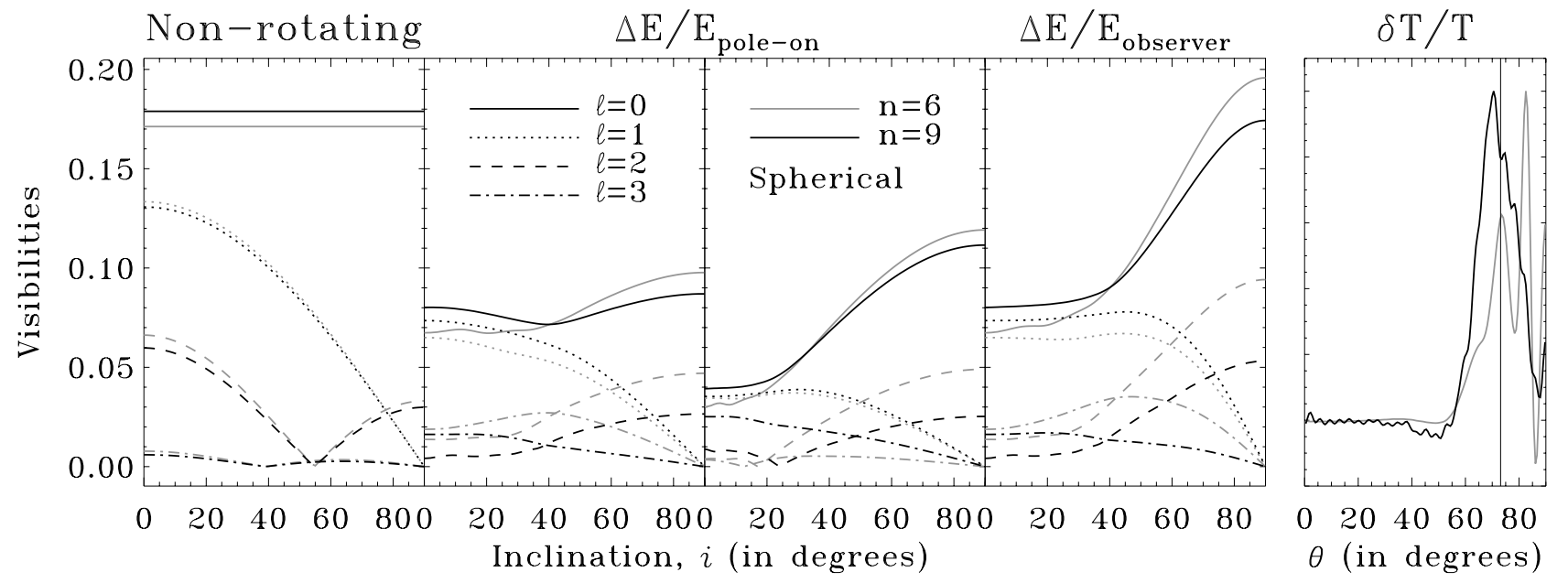

Fig. 10. Visibilities of various island modes as a function of the stellar inclination. The $\ell$ and $n$ values are indicated by the styles and shades of the lines. The rightmost detached panel shows the temperature fluctuations, $\delta T / T$, of the two $\ell=0$ modes in the rotating model.

\subsection{Equatorial concentration}

One of the consequences of the centrifugal force on acoustic modes is the equatorial focusing of island modes around the equator, due to the geometric distortion of the resonant cavity. Lignières et al. (2006) pointed out the possibility that this could lead to higher observed pulsation amplitudes in stars observed in an equator-on configuration, thereby explaining the observations of Suárez et al. (2002), which show a correlation between mode amplitude and inclination. It is also worth noting that for gravito-inertial modes in the inertial regime, modes become trapped in an equatorial waveguide (e.g. Dintrans \& Rieutord 2000; Townsend 2003b). Paradoxically, this usually causes these modes to be more visible in a pole-on configuration, due to the contribution from surface distortion (Townsend 2003a; Daszyńska-Daszkiewicz et al. 2007).

Figure 10 shows the visibilities of various axisymmetric $(m=0)$ acoustic modes as a function of inclination. The left panel corresponds to a non-rotating model, whereas the three middle panels corresponds to a model at $0.8 \Omega_{\mathrm{C}}$. At such a rotation rate, all of the modes in these panels are island modes. The mode visibilities in the second panel are normalised by the pole-on equilibrium luminosity of the star (as is done in Lignières et al. 2006). What is surprising in this figure is that although the symmetric modes 
are more visible from the equator than from the poles, one would expect a higher contrast between the two, especially for the $\ell=0$ modes. Indeed, the surface temperature fluctuations, $\delta T / T$, of the $\ell=0$ modes, depicted in the rightmost detached panel, show a much stronger confinement towards the equatorial region. A careful investigation reveals that the reason for this lack of contrast between polar and equatorial visibilities stems from the geometrical shape of the star. Given the high curvature at the equator, the surface quickly starts pointing more towards a polar direction. To illustrate this point more clearly, the third panel shows the same visibilities assuming the star is spherical, but retaining the other effects of rotation such as gravity darkening. Clearly, a much stronger contrast appears between the poles and the equator. One can also quantify at what latitude a temperature fluctuation contributes more to mode visibilities in an equatorial direction than in a polar one by calculating at what point the ratio,

$R\left(\theta_{0}\right)=\frac{2 \int_{\phi=-\pi / 2}^{\pi / 2} \frac{\partial I}{\partial T_{\mathrm{eff}}}\left[\boldsymbol{n}\left(\theta_{0}, \phi\right) \cdot \boldsymbol{e}_{x}, g_{\mathrm{eff}}\left(\theta_{0}\right), T_{\mathrm{eff}}\left(\theta_{0}\right)\right] \boldsymbol{e}_{x} \cdot \mathrm{d} \boldsymbol{S}}{\int_{\phi=-\pi}^{\pi} \frac{\partial I}{\partial T_{\mathrm{eff}}}\left[\boldsymbol{n}\left(\theta_{0}, \phi\right) \cdot \boldsymbol{e}_{z}, g_{\mathrm{eff}}\left(\theta_{0}\right), T_{\mathrm{eff}}\left(\theta_{0}\right)\right] \boldsymbol{e}_{z} \cdot \mathrm{d} \boldsymbol{S}}$,

exceeds one. In the above formula, only the effects of temperature fluctuations are retained as they usually are the dominant factor in mode visibilities. The denominator corresponds to the intensity fluctuation in the polar direction, $\boldsymbol{e}_{z}$, caused by a unit axisymmetric effective temperature variation at colatitude $\theta_{0}$. The numerator is the intensity fluctuation in the equatorial direction, $\boldsymbol{e}_{x}$, caused by the same unit effective temperature fluctuation, and it's mirror image in the southern hemisphere, at colatitude $\pi-\theta_{0}$, hence the factor 2. In the rotating model used in Fig. 10, the ratio $R\left(\theta_{0}\right)$ exceeds 1 from $73.1^{\circ}$, which is depicted by the vertical line in the rightmost panel. As can be seen in the figure, this line is quite close to the maximum of the $(n, \ell)=(9,0)$ mode, thereby explaining the near equal pole-on and equator-on visibilities. Had the star been spherical, the position of this line would have been $57.0^{\circ}$.

The fourth panel shows the mode visibilities if they are normalised by the equilibrium luminosity in the observer's direction (as is done in Fig. 7). Once more, there is a strong contrast between the polar and equatorial visibilities. This is simply caused by the lower luminosity of the star equator-on, which results from gravity darkening. From an observational point of view, this last ratio is easier to obtain given that $E_{\text {pole-on }}$ is not directly observable, and may be one of the dominant factors in the correlation between inclination and mode amplitude found by Suárez et al. (2002).

\subsection{Avoided crossings}

It is then interesting to look at the effects of avoided crossings on mode visibilities. Lignières et al. (2006) pointed out the possibility that rotationally-induced avoided crossings may explain close frequency pairs observed in $\delta$ Scuti stars (Breger \& Bischof 2002; Breger \& Pamyatnykh 2006). Indeed, the structure of the eigenfunctions of the two (or more) modes involved in an avoided crossing are a mixture of the eigenfunctions prior to the crossing. Hence, if a high-visibility mode interacts with a low-visibility mode, the two may be visible during the crossing when both modes have a mixed character, as was already shown in Daszyńska-Daszkiewicz et al. (2002).

Figure 11 (left panel) shows a set of avoided crossings between families of $\ell=1,5$ and 9 modes, the azimuthal order being $m=1$. As can be seen in the figure, rotation is an ideal way of generating avoided crossings due to its differential effect on modes of different degrees. As was found in Lignières et al. (2006), we also find that modes of degree $\ell$ tend to interact with modes of degree $\ell+4$. The middle panel shows the associated mode visibilities. At $0.48 \Omega_{\mathrm{C}}$, the modes labelled (a) and (b) are strongly interacting, thereby producing an intermediate visibility for both modes. We note that the frequency separation between the two is $2.24 \mu \mathrm{Hz}$, which is slightly larger than for observed close frequency pairs. The meridional cross-section of these modes is depicted in the right panel, both of which show mixed characteristics.

In order for avoided crossings to explain close frequency pairs, a number of conditions need to be meet. On the one hand, the coupling between the modes needs to be sufficiently weak so that the frequency differences become small enough. In the above example, none of the frequencies get closer than $1.4 \mu \mathrm{Hz}$ which is slightly above what is considered to be a close frequency pair. On the other hand, the coupling needs to be sufficiently strong so as to allow the modes to have mixed characteristics over a nonnegligible range in parameter space. Then, one would need to do a full statistical study of the frequency spectrum of visible modes and quantify how many close frequencies are due to avoided crossings and how many result from chance coincidence between uncoupled modes. Such a study is, however, beyond the scope of this paper.

\section{Multi-colour visibilities}

One of the advantages of calculating amplitude ratios between different photometric bands is that the intrinsic amplitude of the mode factors out. As a result, it is possible to predict such ratios from linear theory alone. In this section, we will examine some of the effects of rotation on amplitude ratios. In particular, we will focus on a frequency multiplet, and on modes with the same $\ell$ and $m$ values. However, before discussing different results, it is useful to examine more carefully the question of normalising mode visibilities in multiple photometric bands.

\subsection{Normalisation}

In what follows, we will depart from the traditional approach which consists in normalising the visibilities with respect to a given band. The problem with such an approach is that sometimes, there are modes for which their visibilities nearly cancel out in the chosen band, thereby amplifying their amplitude ratios. This can then exaggerate differences between these ratios and those of other modes. We therefore propose another normalisation scheme which does not favour one band over the others, and which applies to a set of modes which we want to compare. 

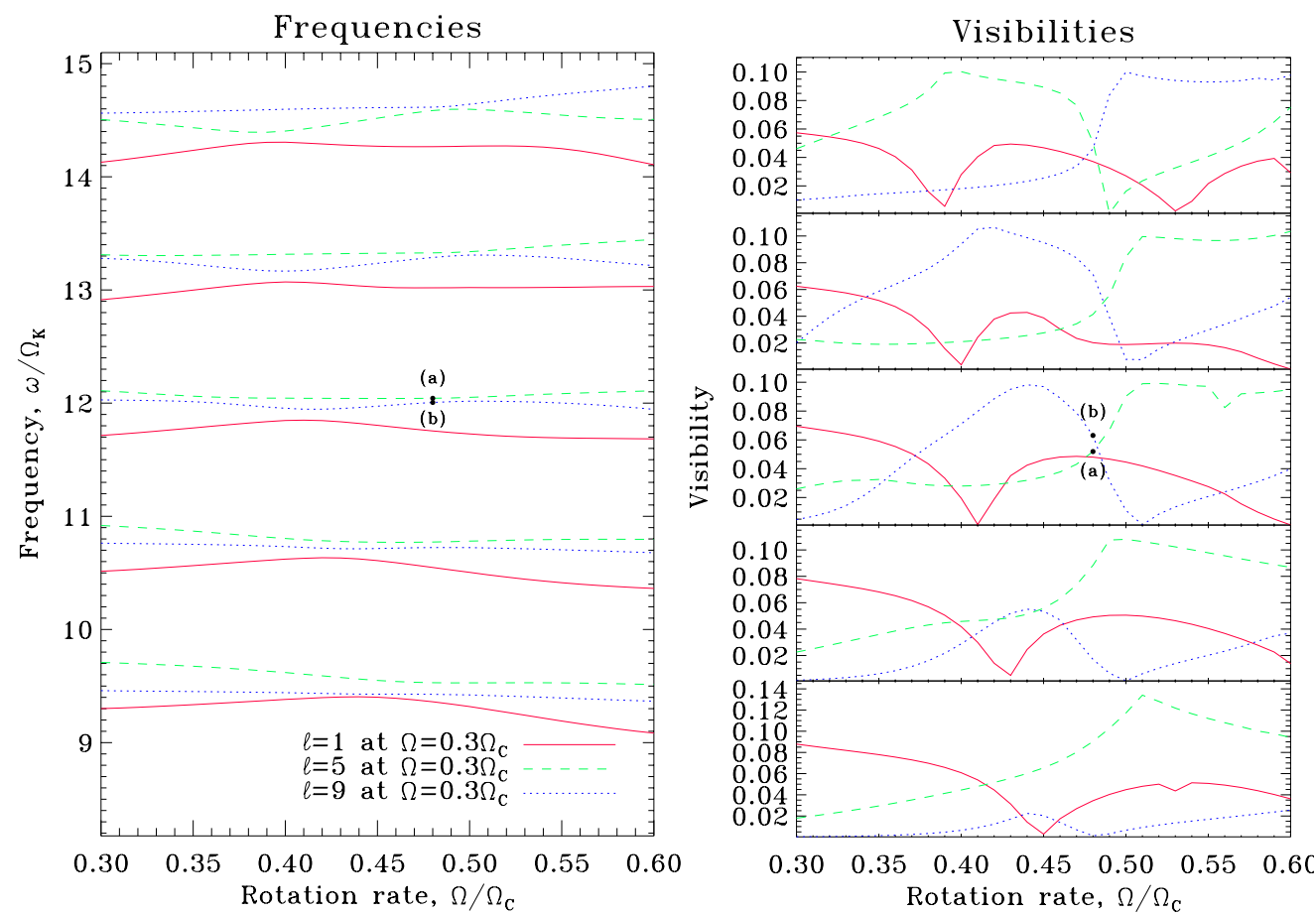

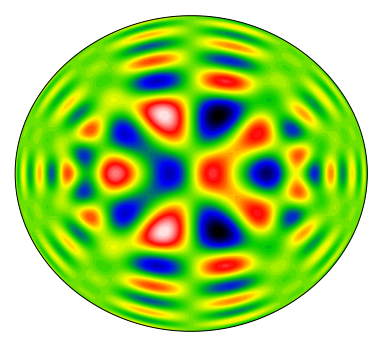

(a) $\nu=728.4 \mu \mathrm{Hz}$

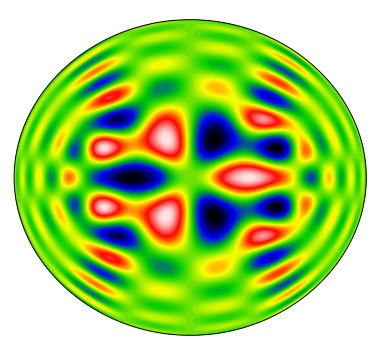

(b) $\nu=726.2 \mu \mathrm{Hz}$

Fig. 11. A set of avoided crossings between $\ell=1,5$ and 9 modes with $m=1$. The linestyles and colours indicate the $\ell$ value at $\Omega=0.3 \Omega_{\mathrm{C}}$ (note that an avoided crossing has already taken place between the uppermost $\ell=5$ and 9 modes, hence the "swapped" identification compared to the other modes). The left panel shows the frequencies, the middle panel gives the visibilities in the CoRoT photometric band for $i=30^{\circ}$ and the right panel shows the meridional cross-sections of two modes undergoing an avoided crossing, and labelled by "(a)" and "(b)" in the two left panels. (This figure is available in color in electronic form.)

We start by considering a set of $N$ modes, numbered $j=1 \ldots N$. Let us denote $V_{i}^{j}$ the visibility of mode $j$ in the photometric band $i$. We will also use the notation $\boldsymbol{V}^{j}$ to represent the vector $\left(V_{i}^{j}\right)_{i=1 \ldots b}$. We want to normalise each mode so as to minimise, in some sense, the distance between their visibilities. To do so, we introduce a set of references visibilities $W_{i}$, which at this point are unknown, and normalise each mode so as to minimise its distance to $W_{i}$. This amounts to minimising the following cost function:

$J=\sum_{j=1}^{N} \sum_{i=1}^{b}\left(A_{j} V_{i}^{j}-W_{i}\right)^{2}$

where $b$ is the number of photometric bands and $A_{j}$ the normalisation factors for each mode. In this expression, both the $A_{j}$ and $W_{i}$ are unknowns. Furthermore, we impose the following constraint, so as to avoid the trivial solution $A_{j} \equiv 0, W_{i} \equiv 0$ :

$\sum_{i=1}^{b} W_{i}^{2}=1$

As will be shown in Appendix D, $\boldsymbol{W}=\left(W_{i}\right)_{i=1 \ldots b}$ turns out to be the principal component of the normalised vectors $\left(\tilde{\boldsymbol{V}}^{j}\right)_{j=1 \ldots N}$, where $\tilde{V}_{i}^{j}=V_{i}^{j} / \sqrt{\sum_{k=1}^{b}\left(V_{k}^{j}\right)^{2}}$. This result is fairly intuitive, since the vectors $\left(\tilde{\boldsymbol{V}}^{j}\right)_{j=1 \ldots N}$ retain the directional information (in $b$ dimensions) associated with each mode while discarding the amplitude (which is arbitrary anyway). Once the reference visibilities are determined, it is straightforward to find the normalisation factors:

$A_{j}=\frac{\sum_{i=1}^{b} V_{i}^{j} W_{i}}{\sum_{i=1}^{b}\left(V_{i}^{j}\right)^{2}}, \quad$ or equivalently, $\quad \tilde{A}_{j}=\sum_{i=1}^{b} \tilde{V}_{i}^{j} W_{i}$.

The normalised visibilities then become $\hat{\boldsymbol{V}}^{j}=A_{j} \boldsymbol{V}^{j}=\tilde{A}_{j} \tilde{\boldsymbol{V}}^{j}$. This last equation has a simple geometrical interpretation: indeed, $\tilde{A}_{j} \tilde{\boldsymbol{V}}^{j}$ represents the projection of $\boldsymbol{W}$ onto the direction $\tilde{\boldsymbol{V}}^{j}$.

In the following sections, we plot normalised visibilities $\hat{\boldsymbol{V}}^{j}$ based on the set of modes relevant to each sub-panel of the various figures, and will call them "amplitude ratios" even if strictly speaking they are not amplitude ratios. In some cases, the reference visibilities, $W_{i}$, are also displayed. It is important to bear in mind that this normalisation depends on the set of modes, and furthermore on their inclination since this affects their visibilities in rotating stars. Hence, the mode-dependant normalisation factors will change from one panel to another, even for the same set of modes, if viewed at different inclinations. Arguably, some of the information 
based on inclination is lost in the process (the same would also be true if we had normalised the visibilities with respect to a given band). However, what is important here is the comparison of amplitude ratios between a set of modes for a given configuration. Furthermore, one does not have the luxury to modify the inclination of an observed star. Another concern is that by normalising the visibilities by a mode-depend scale factor, one is seemingly no longer comparing amplitude ratios which are the true invariants. However, such information is contained within the shape of the curve defined by the normalised visibilities, i.e. the ratios between the components $\left(\hat{V}_{i}^{j}\right)_{i=1 \ldots b}$. Moreover, with the above normalisation, modes with similar amplitude ratios will have similar scale factors, $\tilde{A}_{j}$, and hence normalised visibilities which are similar, even more so than if one of the photometric bands had been used for the purposes of normalisation.

\subsection{A multiplet}

We first start by looking at the $(n, \ell)=(6,2)$ multiplet. Figure 12 shows amplitude ratios, based on the Geneva photometric system, at different rotation rates and inclinations. Only axisymmetric modes are displayed for the pole-on configurations $\left(i=0^{\circ}\right)$ and even modes, i.e. modes which are symmetric with respect to the equator, are shown for the equator-on configurations $\left(i=90^{\circ}\right)$, given that the other modes cancel out. As expected, the amplitude ratios do not depend on the inclination or azimuthal order in the non-rotating case. When the rotation rate increases, this is no longer true, as has been already pointed out by Daszyńska-Daszkiewicz et al. (2002) and Townsend (2003a). It is interesting to note that this departure from a constant behaviour is non-monotonic and therefore, somewhat difficult to predict. For instance, at $(\Omega, i)=\left(0.4 \Omega_{\mathrm{C}}, 30^{\circ}\right)$ the $|m|=2$ modes have higher ratios at lower wavelengths and lower ratios at higher wavelengths. At $(\Omega, i)=\left(0.5 \Omega_{\mathrm{C}}, 30^{\circ}\right)$ the opposite is true. The $|m|=1$ modes have the opposite behaviour. The dependence on orientation seems to be somewhat simpler, although one can note how the mode order frequently changes between $i=30^{\circ}$ and $i=60^{\circ}$. This implies that mode identification from amplitude ratios will not be as straightforward as in the non-rotating case.

It is also interesting to note how the overall shape of most of the ratios change starting from $\Omega=0.6 \Omega_{\mathrm{C}}$. At lower rotation rates, the concavity seems to be pointing up and the largest ratios are obtained at the lowest wavelength. In the most rapidly rotating models, the concavity is pointing downwards, and the ratios at the lowest wavelength are among the smallest. A particularly striking example of a change in behaviour is the drastic modification of the $m=0$ amplitude ratios between $\Omega=0.5 \Omega_{\mathrm{C}}$ and $\Omega=0.6 \Omega_{\mathrm{C}}$, especially for the pole-on configurations $\left(i=0^{\circ}\right)$. In order to gain a better understanding of what is causing this, we plot in Fig. 13 the meridional cross-sections of this mode for a selection of rotation rates. As can be seen, an avoided crossing takes place between $\Omega=0.5 \Omega_{\mathrm{C}}$ and $\Omega=0.6 \Omega_{\mathrm{C}}$. This agrees with Daszyńska-Daszkiewicz et al. (2002) who had also shown that avoided crossings can have an important effect on amplitude ratios and phase differences, and who furthermore showed how these can lead to erroneous identifications. A number of other avoided crossings involving the non-axisymmetric modes also take place around that rotation rate. Another effect which comes into play is the fact that the modes depart from the polar regions to focus around the equator starting from $\Omega \simeq 0.6 \Omega_{\mathrm{C}}$, as can be seen in Fig. 13 for the $m=0$ modes.

One last interesting feature in Fig. 12 is the similarity between prograde and retrograde modes with the same $|m|$ value. Townsend (2003a) had previously noted a similar phenomena for gravito-inertial modes, using the traditional approximation. He was, however, unable to provide an explanation for this behaviour given that the Coriolis force plays a dominant role in the modes he studied, thereby leading to important differences between prograde modes and their retrograde counterparts. The modes shown here are acoustic modes, in which the Coriolis force only plays a minor role. Hence, the structures of prograde modes and their retrograde counterparts are quite similar, thus explaining the similar amplitude ratios.

\subsection{Modes with the same $\ell$ and $m$ values}

Figure 14 shows the amplitude ratios for a set of modes with consecutive radial orders, $n$, and with $(\ell, m)=(1,1)$. In the nonrotating case, amplitude ratios for fixed $(\ell, m)$ values are expected to be similar, though not identical, since the ratios between $\hat{\xi}_{r} / r$, $\delta \hat{T}_{\text {eff }} / T_{\text {eff }}$, and $\delta \hat{g}_{\text {eff }} / g_{\text {eff }}$ can vary slightly from one mode to the next. This is different from the purely geometrically disk-integration factors calculated in Lignières et al. (2006) where strict equality is expected (and obtained) at $\Omega=0$. What is interesting to note here is that for the most part, this similarity between the different ratios continues up to rapid rotation rates, as can be seen in Fig. 14 . The reason for this behaviour is straightforward. Modes with fixed $(\ell, m)$ values produce island modes with the same $(\tilde{\ell}, m)$ values. These modes have a similar lateral structure perpendicular to the underlying ray path, apart from the width which decreases with frequency, and only differ by the number of pseudo-radial nodes. Recently, Pasek et al. (2012) came up with asymptotic expressions for their structure, based on ray-dynamics. One may then wonder why we didn't directly select modes according to their $(\tilde{\ell}, m)$ values. The problem with selecting modes that way is that it mixes together even and odd modes, which have different visibilities when the star is close to equator-on. Although the lateral structure is the same, the underlying ray path curves around the equator so that in odd modes, one of the pseudo-radial nodes actually corresponds to the equator. Hence, even and odd modes with the same $(\tilde{\ell}, m)$ values will have a similar surface structure, if one limits themselves to one of the hemispheres. Accordingly, one set of $(\tilde{\ell}, m)$ values correspond to two different sets of $(\ell, m)$ values, one for even modes and the other for odd modes.

It would, however, be incorrect to conclude that modes with the same $\ell$ and $m$ values systematically lead to similar amplitude ratios. In Fig. 15, we represent the amplitude ratios for modes with $(\ell, m)=(3,0)$. As opposed to Fig. 14, this is one of the worst cases, where agreement is rather poor. Various effects can lead to differences in amplitude ratios. As mentioned above, avoided crossings can cause irregular behaviour. In some cases, a mode can be at the transition where temperature variations start to dominate over surface deformations. This can lead to a very different behaviour from the modes before or after the transition, and cause some of the visibilities to nearly cancel out. A good example of this is the $n=6$ mode for $i=0^{\circ}$ and $\Omega=0.1 \Omega_{\mathrm{C}}$. Finally, if 
D. R. Reese et al.: Mode visibilities in rapidly rotating stars

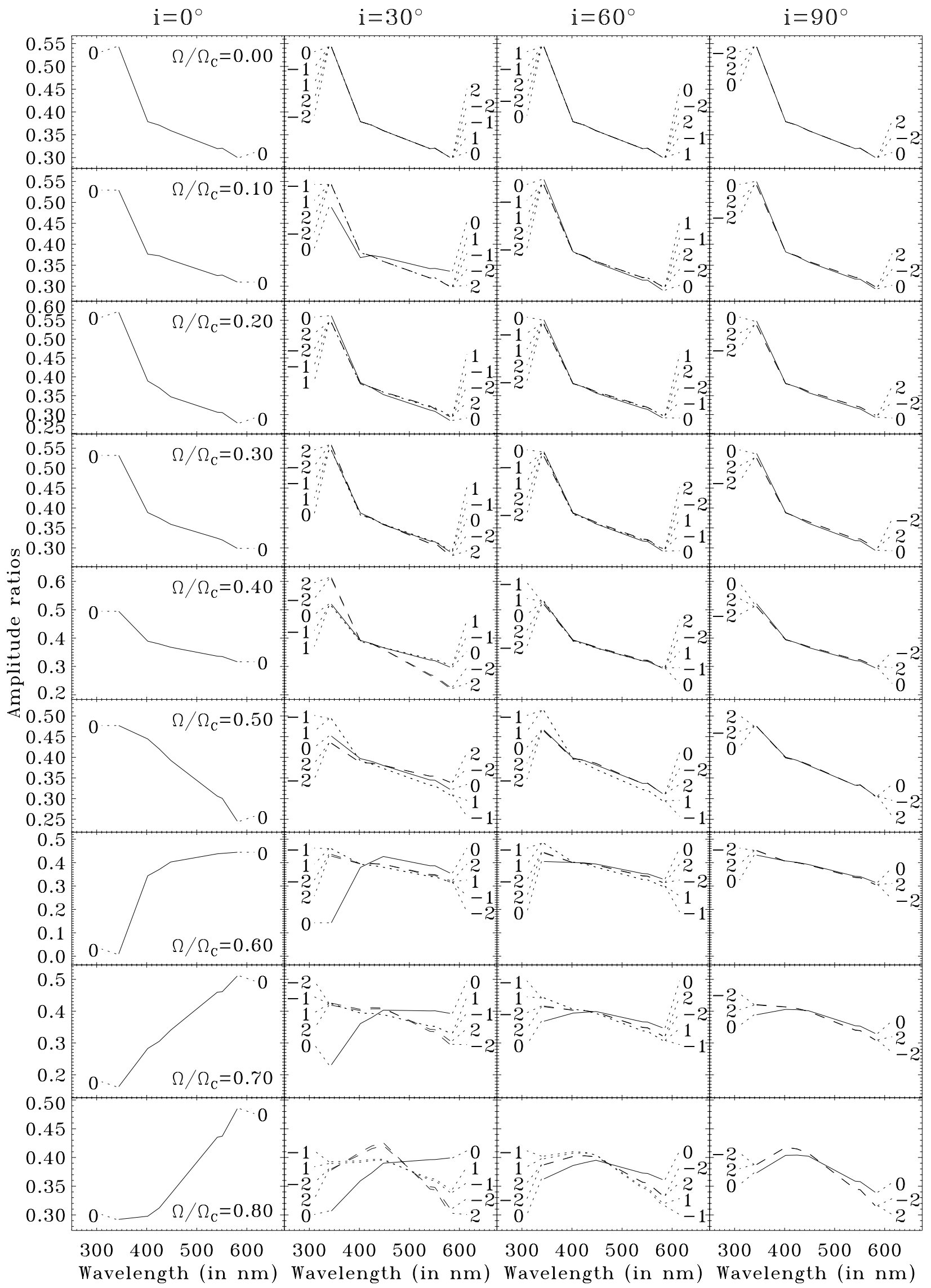

Fig. 12. Amplitude ratios of the $(n, \ell)=(6,2)$ multiplet, based on the Geneva photometric system. Each column corresponds to a different orientation and each row to a different rotation rate. The numbers on either end of each plot and connected by dotted lines indicate the azimuthal order, $m$. The continuous, dotted, and dashed linestyles correspond to $m=0,|m|=1$ and $|m|=2$, respectively. 

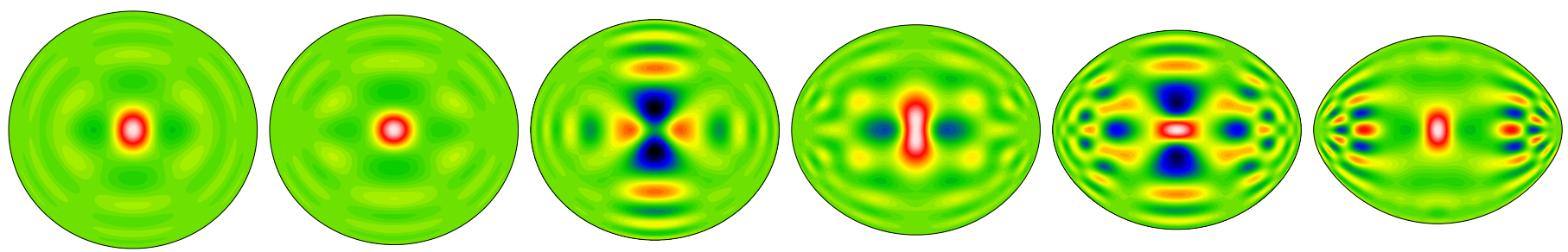

$$
\begin{array}{cc}
\nu=660.1 \mu \mathrm{Hz} & \nu=634.2 \mu \mathrm{Hz} \\
\Omega=0.3 \Omega_{\mathrm{C}} & \Omega=0.4 \Omega_{\mathrm{C}}
\end{array}
$$

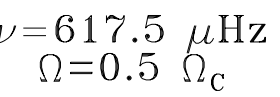

$\nu=585.9 \mu \mathrm{Hz}$

$\nu=548.5 \mu \mathrm{Hz}$

$=501.5 \mu \mathrm{Hz}$

Fig. 13. Meridional cross sections of the $(n, \ell, m)=(6,2,0)$ mode at different rotation rates. At the lowest rotation rates, the modes' structure is dominated by the central parts.

the radial order is too low, a given mode may depart sufficiently from the asymptotic regime to produce noticeable differences on the visibilities.

\section{Conclusion}

In this paper, we derived a new set of equations to calculate mode visibilities in rapidly rotating stars. These equations take into account the centrifugal deformation of the star as well as gravity darkening, and treats the modes in a more realistic way by including the associated effective temperature and gravity variations, as well as the surface distortions. The modes are calculated in fully deformed 2D stellar models based on the SCF method (Jackson et al. 2005; MacGregor et al. 2007), using the 2D oscillation code TOP (Reese et al. 2006, 2009a). A grid of Kurucz atmospheres was used to calculate realistic intensities at each point on the surface (Kurucz 2005). As such, this represents an important step towards obtaining realistic multi-colour visibilities of acoustic modes in rapidly rotating stars, and an improvement over previous studies which approximated the effects of rotation on the model and the oscillations (e.g. Daszyńska-Daszkiewicz et al. 2002; Townsend 2003a) or used simplified visibility calculations (Lignières \& Georgeot 2009).

One of the important limitations in the present study is the use of the adiabatic approximation. Accordingly, the temperature variations are not reliable near the surface and, hence, provide a poor approximation of the effective temperature variations (Dupret et al. 2002). Including non-adiabatic effects is likely to have an important impact on mode visibilities since effective temperature variations often play a dominant role in mode visibilities, as was shown in Fig. 9. Nonetheless, one can hope that the present calculations will give insight into the qualitative behaviour of mode visibilities and amplitude ratios in rapidly rotating stars. In future studies, we plan to implement non-adiabatic effects, including a full treatment of the stellar atmosphere, by applying a similar approach to Dupret et al. (2002). This will require the use of thermally relaxed rapidly rotating models, such as those produced by the ESTER code (Rieutord \& Espinosa Lara 2009; Espinosa Lara 2010).

The results presented here confirm a number of results previously established. For instance, in stars observed pole-on, some of the island modes stand out compared to other modes and form a regular frequency pattern (Lignières \& Georgeot 2009). Consequently, rapidly rotating pulsating stars seen pole-on may be promising asteroseismic targets. Chaotic modes are more visible than their nonrotating counterparts, probably as a result of irregular node placement, thereby complicating the frequency spectrum, especially for equator-on configurations (Lignières \& Georgeot 2009). Avoided crossings can substantially modify mode visibilities and cause modes which are not usually visible to exceed the detection threshold (Daszyńska-Daszkiewicz et al. 2002). Lignières et al. (2006) previously pointed out that these may explain observed close frequency pairs in $\delta$ Scuti stars, although a full statistical study is required to test this possibility. Finally, amplitude ratios depend on both the inclination, $i$, and the azimuthal order, $m$, in rotating stars (Townsend 2003a; Daszyńska-Daszkiewicz et al. 2007). This, of course, makes mode identification in such stars more difficult.

New results were also obtained. In particular, although rapid rotation causes island modes to focus around the equator, the corresponding visibilities show a much smaller contrast between pole-on and equator-on configurations (unless normalised by star's luminosity in the observer's direction). This is because the same geometrical distortion which causes mode focusing in the first place, also increases the proportion of the stellar surface which points in a more poleward direction. We also showed that acoustic modes with the same $(\ell,|m|)$ values tend to have similar amplitude ratios, although this effect is not systematic. The similarity between prograde and retrograde modes stems from the small influence of the Coriolis force on acoustic modes. Modes with the same $(\ell, m)$ values have a similar surface structure, as expected from asymptotic ray theory (Pasek et al. 2012).

In a forthcoming study, we plan to study the statistical properties of the frequency spectra by analysing their autocorrelation functions (Lignières et al. 2010) and by looking at the cumulative distribution functions of the frequency separations. These will be compared with observations, in order to assess up to what extent the present theory is realistic and to see if it is possible to extract global quantities such as the large frequency separation or rotation rate from the observations. Using multi-colour photometry, we will also develop a new strategy for constraining mode identification and obtaining or confirming the values of global quantities.

Acknowledgements. We thank the referee, whose comments helped us clarify the manuscript. We thank M.-A. Dupret, E. Michel, R. Townsend, F. Lignières, and F. Paletou for interesting discussions which have helped to improve this article. We wish to thank S. Jackson, A. Skumanich and T. S. Metcalfe for their contributions to the Self-Consistent Field method, used for generating rotating stellar models. We are very grateful to R. Kurucz for making his ATLAS9 code and opacity data available and open to the scientific community. We wish to thank the KITP at UCSB for their warm hospitality during the research program "Asteroseismology in the Space Age". DRR is financially supported through a postdoctoral fellowship from the "Subside fédéral pour la recherche 2011", University of Liège, and was previously supported by the CNES ("Centre National d'Etudes Spatiales"), both of which are gratefully acknowledged. This work was granted access to the HPC resources of IDRIS under the allocation 2011-99992 made by GENCI ("Grand Equipement National de Calcul Intensif"). The National Center for Atmospheric Research is a federally funded research and development center sponsored by the US National Science Foundation. 
D. R. Reese et al.: Mode visibilities in rapidly rotating stars

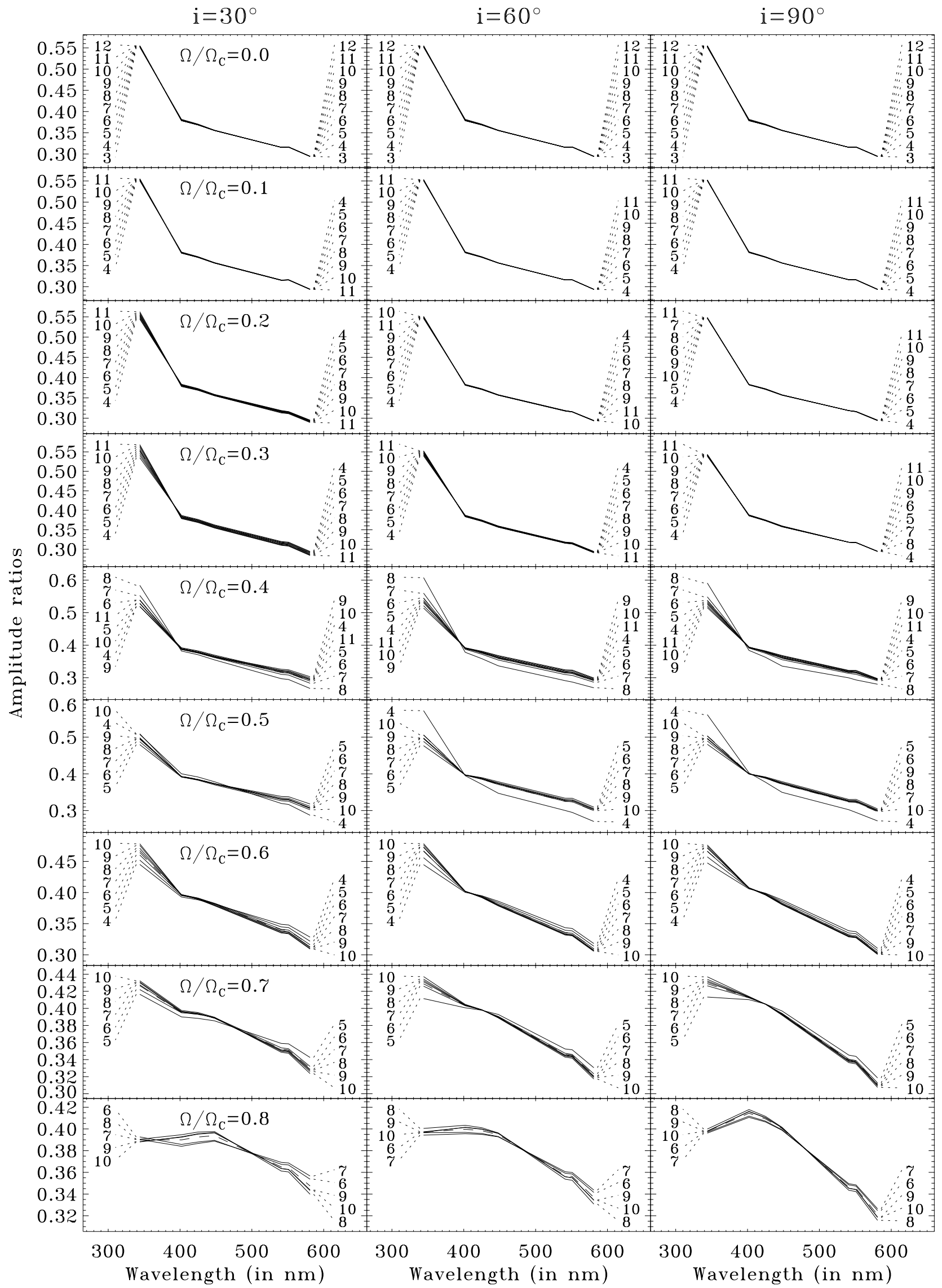

Fig. 14. Amplitude ratios for sets of modes with consecutive radial orders and for which $(\ell, m)=(1,1)$. The reference visibilities used for normalisation, $W_{i}$, are shown as a dashed line in each plot. In most cases, this line is covered up by the amplitude ratios, which are represented by continuous lines. The numbers on either end of the plots indicate the radial orders, $n$. 
A\&A 550, A77 (2013)

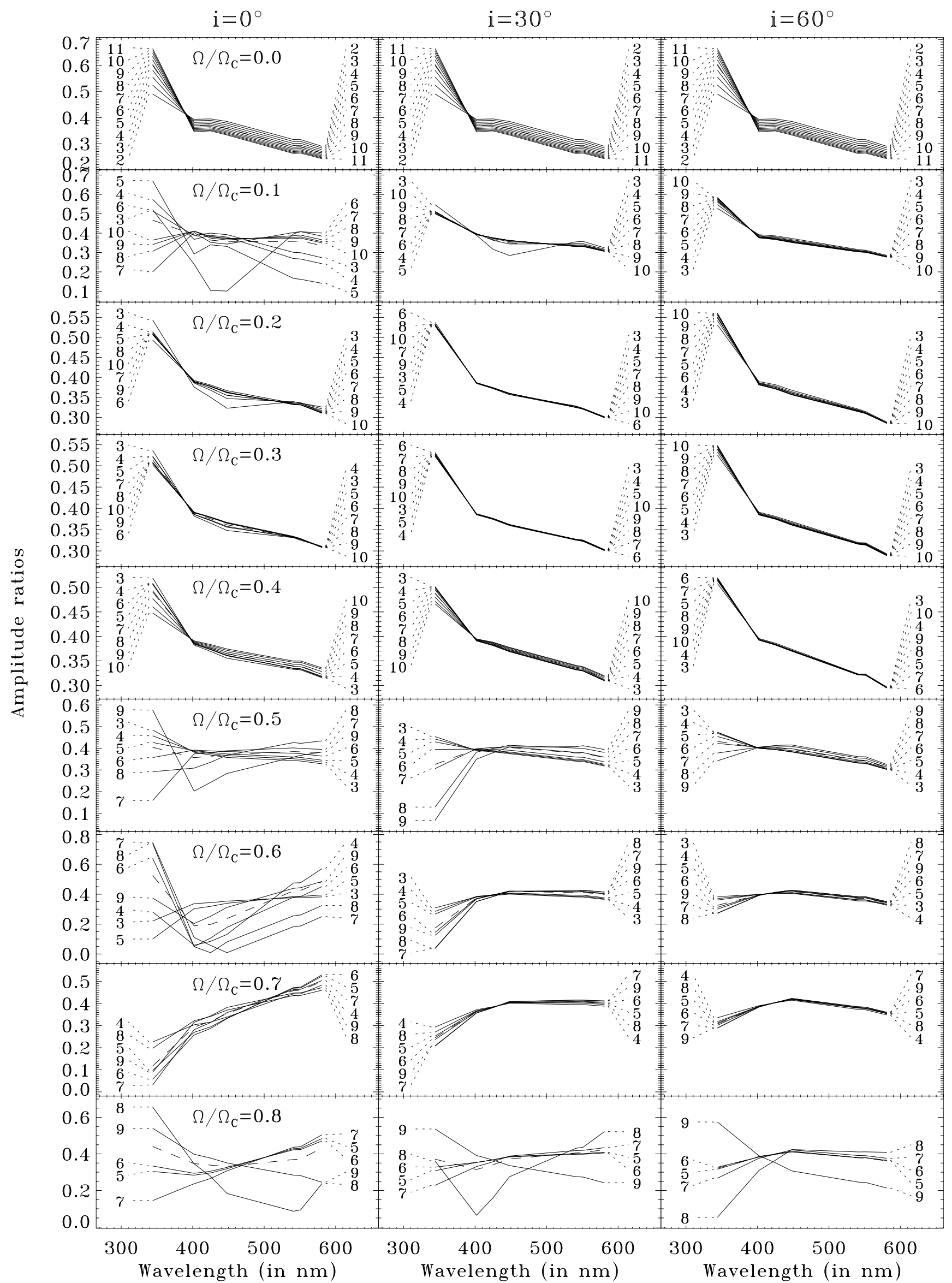

Fig. 15. Same as Fig. 14, except that $(\ell, m)=(3,0)$. 


\section{References}

Auvergne, M., Bodin, P., Boisnard, L., et al. 2009, A\&A, 506, 411

Baglin, A., Auvergne, M., Barge, P., et al. 2009, in IAU Symp., 253, 71

Balona, L. A., Lenz, P., Antoci, V., et al. 2012, MNRAS, 419, 3028

Barban, C., Goupil, M. J., van 't Veer-Menneret, C., et al. 2003, A\&A, 405, 1095

Böhm, T., Lignières, F., Wade, G., et al. 2012, A\&A, 537, A90

Bonazzola, S., Gourgoulhon, E., \& Marck, J.-A. 1998, Phys. Rev. D, 58, 104020

Borucki, W., Koch, D., Batalha, N., et al. 2009, in IAU Symp., 253, 289

Breger, M., \& Bischof, K. M. 2002, A\&A, 385, 537

Breger, M., \& Pamyatnykh, A. A. 2006, MNRAS, 368, 571

Briquet, M., Morel, T., Thoul, A., et al. 2007, MNRAS, 381, 1482

Buta, R. J., \& Smith, M. A. 1979, ApJ, 232, 213

Canuto, V. M., Goldman, I., \& Mazzitelli, I. 1996, ApJ, 473, 550

Claret, A. 2000, A\&A, 363, 1081

Daszyńska-Daszkiewicz, J., Dziembowski, W. A., Pamyatnykh, A. A., \& Goupil, M.-J. 2002, A\&A, 392, 151

Daszyńska-Daszkiewicz, J., Dziembowski, W. A., \& Pamyatnykh, A. A. 2007, Acta Astron., 57, 11

De Ridder, J., Telting, J. H., Balona, L. A., et al. 2004, MNRAS, 351, 324

Dintrans, B., \& Rieutord, M. 2000, A\&A, 354, 86

Dupret, M.-A., De Ridder, J., Neuforge, C., Aerts, C., \& Scuflaire, R. 2002, A\&A, 385, 563

Dupret, M.-A., De Ridder, J., De Cat, P., et al. 2003, A\&A, 398, 677

Dziembowski, W. 1977, Acta Astron., 27, 203

Edmonds, A. R. 1960, Angular Momentum in Quantum Mechanics (Princeton: Princeton University Press)

Eggleton, P. P., Faulkner, J., \& Flannery, B. P. 1973, A\&A, 23, 325

Espinosa Lara, F. 2010, ApSS, 328, 291

García Hernández, A., Moya, A., Michel, E., et al. 2009, A\&A, 506, 79

Heiter, U., Kupka, F., van 't Veer-Menneret, C., et al. 2002, A\&A, 392, 619

Heynderickx, D., Waelkens, C., \& Smeyers, P. 1994, A\&AS, 105, 447

Jackson, S., MacGregor, K. B., \& Skumanich, A. 2005, ApJS, 156, 245

Kurucz, R. 1993, ATLAS9 Stellar Atmosphere Programs and $2 \mathrm{~km} \mathrm{~s}^{-1}$ grid. Kurucz CD-ROM No. 13 (Cambridge, Mass.: Smithsonian Astrophysical Observatory), 13

Kurucz, R. L. 2005, Mem. Soc. Astron. It. Supp., 8, 14

Lignières, F., \& Georgeot, B. 2008, Phys. Rev. E, 78, 016215

Lignières, F., \& Georgeot, B. 2009, A\&A, 500, 1173

Lignières, F., Rieutord, M., \& Reese, D. 2006, A\&A, 455, 607

Lignières, F., Georgeot, B., \& Ballot, J. 2010, Astron. Nachr., 331, 1053

MacGregor, K. B., Jackson, S., Skumanich, A., \& Metcalfe, T. S. 2007, ApJ, 663, 560

Mantegazza, L., Poretti, E., Michel, E., et al. 2012, A\&A, 542, A24

Pasek, M., Lignières, F., Georgeot, B., \& Reese, D. R. 2012, A\&A, 546, A11

Pesnell, W. D. 1990, ApJ, 363, 227

Peterson, D. M., Hummel, C. A., Pauls, T. A., et al. 2006, Nature, 440, 896

Poretti, E., Michel, E., Garrido, R., et al. 2009, A\&A, 506, 85

Reese, D. 2008, J. Phys. Conf. Ser., 118, 012023

Reese, D., Lignières, F., \& Rieutord, M. 2006, A\&A, 455, 621

Reese, D. R., MacGregor, K. B., Jackson, S., Skumanich, A., \& Metcalfe, T. S. 2009a, A\&A, 506, 189

Reese, D. R., Thompson, M. J., MacGregor, K. B., et al. 2009b, A\&A, 506, 183

Rieutord, M., \& Espinosa Lara, F. 2009, Commun. Asteroseismol., 158, 99

Roxburgh, I. W. 2004, A\&A, 428, 171

Suárez, J.-C., Michel, E., Pérez Hernández, F., et al. 2002, A\&A, 390, 523

Townsend, R. H. D. 1997, MNRAS, 284, 839

Townsend, R. H. D. 2003a, MNRAS, 343, 125

Townsend, R. H. D. 2003b, MNRAS, 340, 1020

Zima, W., Wright, D., Bentley, J., et al. 2006, A\&A, 455, 235

Pages 24 to 28 are available in the electronic edition of the journal at http://www . aanda.org 


\section{Appendix A: Equations in spheroidal coordinates}

In this section, we derive explicit expressions for the pulsation equations and the mechanical boundary condition, based on the coordinate system described in Sect. 2.4. However, before giving these expressions, it is useful to recall a few definitions. The natural covariant basis, denoted $\left(\boldsymbol{E}_{\zeta}, \boldsymbol{E}_{\theta}, \boldsymbol{E}_{\phi}\right)$, is defined via the relation $\boldsymbol{E}_{i}=\partial_{i} \boldsymbol{r}$, where $i$ stands for $\zeta, \theta$ or $\phi$, and $\boldsymbol{r}=r \boldsymbol{e}_{r}$ :

$\boldsymbol{E}_{\zeta}=r_{\zeta} \boldsymbol{e}_{r}, \quad \boldsymbol{E}_{\theta}=r_{\theta} \boldsymbol{e}_{r}+r \boldsymbol{e}_{\theta}, \quad \boldsymbol{E}_{\phi}=r \sin \theta \boldsymbol{e}_{\phi}$.

Here $\left(\boldsymbol{e}_{r}, \boldsymbol{e}_{\theta}, \boldsymbol{e}_{\phi}\right)$ is the usual spherical basis associated with the spherical coordinates $(r \theta, \phi)$. The associated dual (contravariant) basis is defined such that $\boldsymbol{E}^{i} \cdot \boldsymbol{E}_{j}=\delta_{j}^{i}$ :

$\boldsymbol{E}^{\zeta}=\frac{\boldsymbol{e}_{r}}{r_{\zeta}}-\frac{r_{\theta} \boldsymbol{e}_{\theta}}{r r_{\zeta}}, \quad \boldsymbol{E}^{\theta}=\frac{\boldsymbol{e}_{\theta}}{r}, \quad \boldsymbol{E}^{\phi}=\frac{\boldsymbol{e}_{\phi}}{r \sin \theta}$.

The vector $\boldsymbol{E}^{\zeta}$ is perpendicular to surfaces of constant $\zeta$ value, including the stellar surface. As in Reese et al. (2006), we derive an alternate basis from $\left(\boldsymbol{E}_{\zeta}, \boldsymbol{E}_{\theta}, \boldsymbol{E}_{\phi}\right)$ as follows:

$\boldsymbol{a}_{\zeta}=\frac{\zeta^{2}}{r^{2} r_{\zeta}} \boldsymbol{E}_{\zeta}, \quad \boldsymbol{a}_{\theta}=\frac{\zeta}{r^{2} r_{\zeta}} \boldsymbol{E}_{\theta}, \quad \boldsymbol{a}_{\phi}=\frac{\zeta}{r^{2} r_{\zeta} \sin \theta} \boldsymbol{E}_{\phi}$

In the spherical limit, the alternate basis converges to the spherical basis. The Lagrangian displacement is decomposed over the alternate basis as follows:

$\boldsymbol{\xi}=\boldsymbol{\xi}^{\zeta} \boldsymbol{a}_{\zeta}+\xi^{\theta} \boldsymbol{a}_{\theta}+\xi^{\phi} \boldsymbol{a}_{\phi}$

These components are related to the spherical components (see Eq. (20)) as follows:

$\xi_{r}=\frac{\zeta^{2}}{r^{2}} \xi^{\zeta}+\frac{\zeta r_{\theta}}{r^{2} r_{\zeta}} \xi^{\theta}, \quad \xi_{\theta}=\frac{\zeta}{r r_{\zeta}} \xi^{\theta}, \quad \xi_{\phi}=\frac{\zeta}{r r_{\zeta}} \xi^{\phi}$,

where superscripts are used with the alternate components, and subscripts with the spherical components. Based on the alternate components, the dot product $\boldsymbol{\xi} \cdot \boldsymbol{g}_{\text {eff }}$ becomes:

$\boldsymbol{\xi} \cdot \boldsymbol{g}_{\mathrm{eff}}=\frac{\zeta^{2}}{r^{2} r_{\zeta}} \frac{\partial_{\zeta} P_{0}}{\rho_{0}} \xi^{\zeta}+\frac{\zeta}{r^{2} r_{\zeta}} \frac{\partial_{\theta} P_{0}}{\rho_{0}} \xi^{\theta}=\mathcal{G}_{\zeta} \xi^{\zeta}+\mathcal{G}_{\theta} \xi^{\theta}$,

where we have introduced the following quantities:

$\mathcal{G}_{\zeta}=\frac{\zeta^{2}}{r^{2} r_{\zeta}} \frac{\partial_{\zeta} P_{0}}{\rho_{0}}, \quad \mathcal{G}_{\theta}=\frac{\zeta}{r^{2} r_{\zeta}} \frac{\partial_{\theta} P_{0}}{\rho_{0}}$.

\section{A.1. Pulsation equations}

We now give explicit expressions for the pulsation equations in spheroidal coordinates. The continuity equation is:

$0=\frac{\delta \rho}{\rho_{0}}+\frac{\zeta^{2}}{r^{2} r_{\zeta}}\left[\frac{\partial_{\zeta}\left(\zeta^{2} \xi^{\zeta}\right)}{\zeta^{2}}+\frac{\partial_{\theta}\left(\sin \theta \xi^{\theta}\right)}{\zeta \sin \theta}+\frac{\partial_{\phi} \xi^{\phi}}{\zeta \sin \theta}\right]$.

Euler's equation takes on the following form:

$$
\begin{aligned}
0= & {[\omega+m \Omega]^{2}\left[\frac{\zeta^{2} r_{\zeta} \xi^{\zeta}}{r^{2}}+\frac{\zeta r_{\theta} \xi^{\theta}}{r^{2}}\right]+2 i[\omega+m \Omega] \frac{\Omega \zeta \sin \theta}{r} \xi^{\phi}-s\left(\partial_{s} \Omega^{2}\right) r_{\zeta} \sin \theta\left[\frac{\zeta^{2} \sin \theta}{r^{2}} \xi^{\zeta}+\frac{\zeta\left(r_{\theta} \sin \theta+r \cos \theta\right)}{r^{2} r_{\zeta}} \xi^{\theta}\right] } \\
& -\frac{P_{0}}{\rho_{0}} \partial_{\zeta}\left(\frac{\delta p}{P_{0}}\right)+\frac{\partial_{\zeta} P_{0}}{\rho_{0}}\left(\frac{\delta \rho}{\rho_{0}}-\frac{\delta p}{P_{0}}\right)-\partial_{\zeta} \Psi+\left(\partial_{\zeta} \mathcal{G}_{\zeta}\right) \xi^{\zeta}+\mathcal{G}_{\zeta} \partial_{\zeta} \xi^{\zeta}+\left(\partial_{\zeta} \mathcal{G}_{\theta}\right) \xi^{\theta}+\mathcal{G}_{\theta} \partial_{\zeta} \xi^{\theta}, \\
0= & {[\omega+m \Omega]^{2}\left[\frac{\zeta^{2} r_{\theta} \xi^{\zeta}}{r^{2}}+\frac{\zeta\left(r^{2}+r_{\theta}^{2}\right) \xi^{\theta}}{r^{2} r_{\zeta}}\right]+2 i[\omega+m \Omega] \frac{\Omega \zeta\left(r_{\theta} \sin \theta+r \cos \theta\right)}{r r_{\zeta}} \xi^{\phi} } \\
& -s\left(\partial_{s} \Omega^{2}\right)\left(r_{\theta} \sin \theta+r \cos \theta\right)\left[\frac{\zeta^{2} \sin \theta}{r^{2}} \xi^{\zeta}+\frac{\zeta\left(r_{\theta} \sin \theta+r \cos \theta\right)}{r^{2} r_{\zeta}} \xi^{\theta}\right] \\
& -\frac{P_{0}}{\rho_{0}} \partial_{\theta}\left(\frac{\delta p}{P_{0}}\right)+\frac{\partial_{\theta} P_{0}}{\rho_{0}}\left(\frac{\delta \rho}{\rho_{0}}-\frac{\delta p}{P_{0}}\right)-\partial_{\theta} \Psi+\left(\partial_{\theta} \mathcal{G}_{\zeta}\right) \xi^{\zeta}+\mathcal{G}_{\zeta} \partial_{\theta} \xi^{\zeta}+\left(\partial_{\theta} \mathcal{G}_{\theta}\right) \xi^{\theta}+\mathcal{G}_{\theta} \partial_{\theta} \xi^{\theta}, \\
0= & {[\omega+m]^{2} \frac{\zeta}{r_{\zeta}} \xi^{\phi}-2 i[\omega+m \Omega] \frac{\Omega^{2} \sin \theta}{r} \xi^{\zeta}-2 i[\omega+m \Omega] \frac{\Omega \zeta\left(r_{\theta} \sin \theta+r \cos \theta\right)}{r r_{\zeta}} \xi^{\theta} } \\
& -\frac{P_{0}}{\rho_{0}} \frac{\partial_{\phi}}{\sin \theta}\left(\frac{\delta p}{P_{0}}\right)-\frac{\partial_{\phi} \Psi}{\sin \theta}+\mathcal{G}_{\zeta} \frac{\partial_{\phi} \xi^{\zeta}}{\sin \theta}+\mathcal{G}_{\theta} \frac{\partial_{\phi} \xi^{\theta}}{\sin \theta},
\end{aligned}
$$

A77, page 24 of 28 
where $s=r \sin \theta$ is the distance to the rotation axis. Poisson's equation becomes:

$0=\frac{r^{2}+r_{\theta}^{2}}{r^{2} r_{\zeta}^{2}} \partial_{\zeta \zeta}^{2} \Psi+c_{\zeta} \partial_{\zeta} \Psi-\frac{2 r_{\theta}}{r^{2} r_{\zeta}} \partial_{\zeta \theta}^{2} \Psi+\frac{1}{r^{2}} \Delta_{\theta \phi} \Psi-4 \pi\left\{\rho_{0} \frac{\delta \rho}{\rho_{0}}-\frac{\zeta^{2}}{r^{2} r_{\zeta}}\left(\partial_{\zeta} \rho_{0} \xi^{\zeta}+\frac{\partial_{\theta} \rho_{0} \xi^{\theta}}{\zeta}\right)\right\}$,

where

$$
\begin{aligned}
c_{\zeta} & =\frac{1}{r^{2} r_{\zeta}^{3}}\left(2 r_{\zeta} r_{\theta} r_{\zeta \theta}-r^{2} r_{\zeta \zeta}-r_{\zeta}^{2} r_{\theta \theta}+2 r r_{\zeta}^{2}-r_{\theta}^{2} r_{\zeta \zeta}-r_{\zeta}^{2} r_{\theta} \cot \theta\right), \\
\Delta_{\theta \phi} & =\partial_{\theta \theta}^{2}+\cot \theta \partial_{\theta}+\frac{1}{\sin ^{2} \theta} \partial_{\phi \phi}^{2} .
\end{aligned}
$$

As was pointed out in Sect. 2.2, the relative Lagrangian density perturbation, $\delta \rho / \rho_{0}$, can be eliminated in favour of the relative Lagrangian pressure perturbation, $\delta p / P_{0}$, thanks to the adiabatic relation, Eq. (6).

\section{A.2. Mechanical boundary condition}

As explained in Sect. 2.5, the mechanical boundary condition is obtained by calculating the dot product between $\boldsymbol{E}^{\zeta}$ and Euler's equation, and cancelling out the vertical gradient of $\delta p / P_{0}$. Furthermore, the quantity $\partial_{\zeta} \xi^{\zeta}$ is eliminated through the continuity equation, and the terms $\mathcal{G}_{\theta}$ and $\partial_{\theta} \mathcal{G}_{\theta}$ vanish at the surface. In spheroidal components, one obtains:

$$
\begin{aligned}
0= & {[\omega+m \Omega]^{2} \frac{\zeta^{2}}{r^{2} r_{\zeta}} \xi^{\zeta}+2 i[\omega+m \Omega] \Omega \frac{\zeta\left(r \sin \theta-r_{\theta} \cos \theta\right)}{r^{2} r_{\zeta}^{2}} \xi^{\phi}-s \partial_{s}\left(\Omega^{2}\right) \frac{r \sin \theta-r_{\theta} \cos \theta}{r r_{\zeta}}\left(\frac{\zeta^{2} \sin \theta}{r^{2}} \xi^{\zeta}+\frac{\zeta\left(r_{\theta} \sin \theta+r \cos \theta\right)}{r^{2} r_{\zeta}} \xi^{\theta}\right) } \\
& +\left(\frac{r^{2}+r_{\theta}^{2}}{r^{2} r_{\zeta}^{2}} \frac{\partial_{\zeta} P_{0}}{\rho_{0}}-\frac{r_{\theta}}{r^{2} r_{\zeta}} \frac{\partial_{\theta} P_{0}}{\rho_{0}}\right)\left(\frac{\delta \rho}{\rho_{0}}-\frac{\delta p}{P_{0}}\right)-\frac{r^{2}+r_{\theta}^{2}}{r^{2} r_{\zeta}^{2}} \partial_{\zeta} \Psi+\frac{r_{\theta}}{r^{2} r_{\zeta}} \partial_{\theta} \Psi-\frac{r^{2}+r_{\theta}^{2}}{\zeta^{2} r_{\zeta}} \mathcal{G}_{\zeta} \frac{\delta \rho}{\rho_{0}}+\frac{r^{2}+r_{\theta}^{2}}{r^{2} r_{\zeta}^{2}}\left(\partial_{\zeta} \mathcal{G}_{\zeta}-\frac{2 \mathcal{G}_{\zeta}}{\zeta}\right) \xi^{\zeta} \\
& -\frac{r_{\theta}}{r^{2} r_{\zeta}}\left[\left(\partial_{\theta} \mathcal{G}_{\zeta}\right) \xi^{\zeta}+\mathcal{G}_{\zeta} \partial_{\theta} \xi^{\zeta}\right]+\frac{r^{2}+r_{\theta}^{2}}{r^{2} r_{\zeta}^{2}}\left[\left(\partial_{\zeta} \mathcal{G}_{\theta}\right) \xi^{\theta}-\mathcal{G}_{\zeta} \frac{\partial_{\theta}\left(\sin \theta \xi^{\theta}\right)}{\zeta \sin \theta}-\mathcal{G}_{\zeta} \frac{\partial_{\phi} \xi^{\phi}}{\zeta \sin \theta}\right]
\end{aligned}
$$

\section{Appendix B: Lagrangian perturbation to the effective gravity}

As was explained in Sect. 3.2, the Lagrangian perturbation to the effective gravity, $\delta g_{\text {eff }}$, is deduced from the vectorial Lagrangian perturbation to the effective gravity, $\delta \boldsymbol{g}_{\text {eff }}$, via the relation $\delta g_{\text {eff }}=-\boldsymbol{n} \cdot \delta \boldsymbol{g}_{\text {eff }}$, where $\boldsymbol{n}$ is the outward normal at the surface. Furthermore, $\delta \boldsymbol{g}_{\text {eff }}$ includes the Lagrangian perturbation to the gradient of the gravitational potential and the acceleration of a particle tied to the surface, resulting from the oscillatory motions. After adding and subtracting $\boldsymbol{\xi} \cdot \boldsymbol{\nabla}\left(s \Omega^{2} \boldsymbol{e}_{s}\right)$ in order to introduce the equilibrium effective gravity, a vectorial expression is obtained in Eq. (34), and is reproduced here for convenience:

$\delta \boldsymbol{g}_{\mathrm{eff}}=-\boldsymbol{\nabla} \Psi+\boldsymbol{\xi} \cdot \boldsymbol{\nabla} \boldsymbol{g}_{\mathrm{eff}}+(\omega+m \boldsymbol{\Omega})^{2} \boldsymbol{\xi}-2 i(\omega+m \Omega) \boldsymbol{\Omega} \times \boldsymbol{\xi}-\boldsymbol{\Omega} \times(\boldsymbol{\Omega} \times \boldsymbol{\xi})-\boldsymbol{\xi} \cdot \boldsymbol{\nabla}\left(s \Omega^{2} \boldsymbol{e}_{s}\right)$.

In what follows, we will go through the above equation one term at a time in order to obtain explicit expressions for the dot product of $\boldsymbol{n}$ with each one.

The Eulerian perturbation to gravity is obtained through tensor analysis:

$-\boldsymbol{n} \cdot \boldsymbol{\nabla} \Psi=-\frac{r r_{\zeta} \boldsymbol{E}^{\zeta} \cdot\left(\partial_{i} \Psi\right) \boldsymbol{E}^{i}}{\left(r^{2}+r_{\theta}^{2}\right)^{1 / 2}}=-\frac{r r_{\zeta} g^{\zeta i} \partial_{i} \Psi}{\left(r^{2}+r_{\theta}^{2}\right)^{1 / 2}}=-\frac{\left(r^{2}+r_{\theta}^{2}\right)^{1 / 2}}{r r_{\zeta}} \partial_{\zeta} \Psi+\frac{r_{\theta}}{r\left(r^{2}+r_{\theta}^{2}\right)^{1 / 2}} \partial_{\theta} \Psi$

where we have used the relation $\boldsymbol{n}=\frac{r r_{\zeta}}{\left(r^{2}+r_{\theta}^{1}\right)^{1 / 2}} \boldsymbol{E}^{\zeta}$. Furthermore, we have used Einstein's summation convention on repeated indices.

Before dealing with the next term, it is useful to introduce the contravariant components of the Lagrangian displacement, which we distinguish from the components given in Eq. (A.4) by placing a tilde over the top:

$\boldsymbol{\xi}=\tilde{\xi}^{\zeta} \boldsymbol{E}_{\zeta}+\tilde{\xi}^{\theta} \boldsymbol{E}_{\theta}+\tilde{\xi}^{\phi} \boldsymbol{E}_{\phi}$.

where $\left(\boldsymbol{E}_{\zeta}, \boldsymbol{E}_{\theta}, \boldsymbol{E}_{\phi}\right)$ is given in Eq. (A.1). We also introduce the covariant components of the effective gravity:

$\boldsymbol{g}_{\text {eff }}=-g_{\zeta}^{\text {eff }} \boldsymbol{E}^{\zeta}-g_{\theta}^{\text {eff }} \boldsymbol{E}^{\theta}$.

From Eq. (31), it is straightforward to see that $g_{i}^{\text {eff }}=-\partial_{i} P_{0} / \rho_{0}$. Furthermore, $g_{\zeta}^{\text {eff }}=\frac{r r_{\zeta}}{\left(r^{2}+r_{\theta}^{2}\right)^{1 / 2}} g_{\text {eff }}$ and $g_{\theta}^{\text {eff }} \equiv 0$ at the stellar surface.

In tensorial notation, the term $\boldsymbol{n} \cdot\left\{\boldsymbol{\xi} \cdot \boldsymbol{\nabla} \boldsymbol{g}_{\text {eff }}\right\}$ becomes:

$\boldsymbol{n} \cdot\left\{\boldsymbol{\xi} \cdot \boldsymbol{\nabla} \boldsymbol{g}_{\mathrm{eff}}\right\}=\frac{r r_{\zeta}}{\left(r^{2}+r_{\theta}^{2}\right)^{1 / 2}} \boldsymbol{E}^{\zeta} \cdot\left\{\boldsymbol{\xi} \cdot \boldsymbol{\nabla} \boldsymbol{g}_{\mathrm{eff}}\right\}=-\frac{r r_{\zeta}}{\left(r^{2}+r_{\theta}^{2}\right)^{1 / 2}} \tilde{\xi}^{i}\left(\partial_{i} g_{j}^{\mathrm{eff}}-\Gamma_{i j}^{k} g_{k}^{\mathrm{eff}}\right) g^{\zeta j}$ 
where $g^{i j}=\boldsymbol{E}^{i} \cdot \boldsymbol{E}^{j}$ denotes the contravariant components of the metric tensor, and $\Gamma_{i j}^{k}=\left(\partial_{i} \boldsymbol{E}_{j}\right) \cdot \boldsymbol{E}^{k}=-\left(\partial_{i} \boldsymbol{E}^{k}\right) \cdot \boldsymbol{E}_{j}$ the Christoffel coefficients. Equation (B.5) can be simplified if we use the following relation:

$\boldsymbol{\nabla} \cdot \boldsymbol{g}_{\mathrm{eff}}=-g^{i j}\left(\partial_{i} g_{j}^{\mathrm{eff}}-\Gamma_{i j}^{k} g_{k}^{\mathrm{eff}}\right)=-4 \pi G \rho_{0}+\boldsymbol{\nabla} \cdot\left(s \Omega^{2} \boldsymbol{e}_{s}\right)$,

which is a modified form of Poisson's equation. The result is:

$\boldsymbol{n} \cdot\left\{\boldsymbol{\xi} \cdot \nabla \boldsymbol{g}_{\mathrm{eff}}\right\}=\frac{r r_{\zeta}}{\left(r^{2}+r_{\theta}^{2}\right)^{1 / 2}}\left\{\tilde{\xi}^{\zeta}\left[-4 \pi G \rho_{0}+\nabla \cdot\left(s \Omega^{2} \boldsymbol{e}_{s}\right)\right]+\tilde{\xi}^{\zeta}\left(g^{\zeta \theta} \partial_{\theta} g_{\zeta}^{\mathrm{eff}}-A g_{\zeta}^{\mathrm{eff}}\right)+\tilde{\xi}^{\theta}\left(-g^{\zeta \zeta} \partial_{\theta} g_{\zeta}^{\mathrm{eff}}+B g_{\zeta}^{\mathrm{eff}}\right)\right\}$

where

$A=g^{\zeta \theta} \Gamma_{\zeta \theta}^{\zeta}+g^{\theta \theta} \Gamma_{\theta \theta}^{\zeta}+g^{\phi \phi} \Gamma_{\phi \phi}^{\zeta}=\frac{-2 r^{2} r_{\zeta}-r_{\zeta} r_{\theta}^{2}+r r_{\zeta} r_{\theta \theta}-r r_{\theta} r_{\zeta \theta}+r r_{\zeta} r_{\theta} \cot \theta}{r^{3} r_{\zeta}^{2}}$

$B=g^{\zeta \zeta} \Gamma_{\zeta \theta}^{\zeta}+g^{\zeta \theta} \Gamma_{\theta \theta}^{\zeta}=\frac{r_{\zeta} r_{\theta}^{3}+r^{3} r_{\zeta \theta}+r r_{\theta}^{2} r_{\zeta \theta}-r r_{\zeta} r_{\theta} r_{\theta \theta}}{r^{3} r_{\zeta}^{3}}$,

and where we have made use of the following simplifications: $g_{\theta}^{\text {eff }}=\partial_{\theta} g_{\theta}^{\text {eff }}=0$ on the surface, $g^{i j}=g^{j i}$ and $\Gamma_{i j}^{k}=\Gamma_{j i}^{k}$. The above expression can then be re-expressed in terms of $\xi_{r}, \xi_{\theta}$ and $g_{\text {eff }}$ to yield:

$\begin{aligned} \boldsymbol{n} \cdot\left\{\boldsymbol{\xi} \cdot \boldsymbol{\nabla} \boldsymbol{g}_{\mathrm{eff}}\right\}= & \frac{r \xi_{r}-r_{\theta} \xi_{\theta}}{\left(r^{2}+r_{\theta}^{2}\right)^{1 / 2}}\left\{-4 \pi G \rho_{0}+\boldsymbol{\nabla} \cdot\left(s \Omega^{2} \boldsymbol{e}_{s}\right)\right\}+\xi_{r}\left\{-\frac{r_{\theta} \partial_{\theta} g_{\mathrm{eff}}}{r^{2}+r_{\theta}^{2}}+\frac{\left(2 r-r_{\theta} \cot \theta\right)\left(r^{2}+r_{\theta}^{2}\right)+r r_{\theta}^{2}-r^{2} r_{\theta \theta}}{\left(r^{2}+r_{\theta}^{2}\right)^{2}} g_{\mathrm{eff}}\right\} \\ & +\xi_{\theta}\left\{-\frac{r \partial_{\theta} g_{\mathrm{eff}}}{r^{2}+r_{\theta}^{2}}+\frac{r r_{\theta}\left(-2 r^{2}-3 r_{\theta}^{2}+r r_{\theta \theta}\right)+\left(r^{2}+r_{\theta}^{2}\right) r_{\theta}^{2} \cot \theta}{r\left(r^{2}+r_{\theta}^{2}\right)^{2}} g_{\mathrm{eff}}\right\} .\end{aligned}$

The term $\boldsymbol{\nabla} \cdot\left(s \Omega^{2} \boldsymbol{e}_{s}\right)$ takes on the following expression for a general rotation profile, $\Omega \equiv \Omega(\zeta, \theta)$ :

$\boldsymbol{\nabla} \cdot\left(s \Omega^{2} \boldsymbol{e}_{s}\right)=2 \Omega^{2}+\frac{\sin \theta\left(r \sin \theta-r_{\theta} \cos \theta\right)}{r_{\zeta}} \partial_{\zeta}\left(\Omega^{2}\right)+\sin \theta \cos \theta \partial_{\theta}\left(\Omega^{2}\right)$.

For a cylindrical rotation profile, $\Omega \equiv \Omega(s)$, it becomes:

$\boldsymbol{\nabla} \cdot\left(s \Omega^{2} \boldsymbol{e}_{s}\right)=2 \Omega^{2}+s \partial_{s}\left(\Omega^{2}\right)$.

The term $(\omega+m \Omega)^{2} \boldsymbol{n} \cdot \boldsymbol{\xi}$ is given by:

$(\omega+m \Omega)^{2} \boldsymbol{n} \cdot \boldsymbol{\xi}=(\omega+m \Omega)^{2} \frac{r \xi_{r}-r_{\theta} \xi_{\theta}}{\left(r^{2}+r_{\theta}^{2}\right)^{1 / 2}}$.

The Coriolis term is given by:

$-2 \mathrm{i}(\omega+m \Omega) \boldsymbol{n} \cdot(\boldsymbol{\Omega} \times \boldsymbol{\xi})=2 \mathrm{i}(\omega+m \Omega) \Omega \frac{\left(r \sin \theta-r_{\theta} \cos \theta\right) \xi_{\phi}}{\left(r^{2}+r_{\theta}^{2}\right)^{1 / 2}}$.

The last two terms are more conveniently treated together. They take on the following expression for a general rotation profile:

$-\boldsymbol{\Omega} \times(\boldsymbol{\Omega} \times \boldsymbol{\xi})-\boldsymbol{\xi} \cdot \boldsymbol{\nabla}\left(s \Omega^{2} \boldsymbol{e}_{s}\right)=-\left[\frac{r \xi_{r}-r_{\theta} \xi_{\theta}}{r_{\zeta}} \sin \theta \partial_{\zeta}\left(\Omega^{2}\right)+\xi_{\theta} \sin \theta \partial_{\theta}\left(\Omega^{2}\right)\right] \boldsymbol{e}_{s}$

If the rotation profile is cylindrical, they become:

$-\boldsymbol{\Omega} \times(\boldsymbol{\Omega} \times \boldsymbol{\xi})-\boldsymbol{\xi} \cdot \boldsymbol{\nabla}\left(s \Omega^{2} \boldsymbol{e}_{s}\right)=-\left(\sin \theta \xi_{r}+\cos \theta \xi_{\theta}\right) s \partial_{s}\left(\Omega^{2}\right) \boldsymbol{e}_{s}$.

Combining all of these equations together, and remembering the minus sign, yields Eq. (35).

A77, page 26 of 28 


\section{Appendix C: Cancelling of simplified disk-integration factors}

Given the simplified form of the disk-integration factors given in Eq. (45) (see Sect. 3.7), it turns out that some of these cancel out regardless of inclination. To see this, one needs to start with an explicit form for Eq. (45):

$D(i) \cos (\omega t+\psi)=\frac{\mathfrak{R}\left\{\int_{\theta=0}^{\pi} \int_{\phi=-f(\theta)}^{f(\theta)} \delta \hat{T}(\theta) \mathrm{e}^{\mathrm{i} m \phi+i \omega t}\left[r(\sin i \sin \theta \cos \phi+\cos i \cos \theta)-r_{\theta}(\sin i \cos \theta \cos \phi-\cos i \sin \theta)\right] r \sin \theta \mathrm{d} \theta \mathrm{d} \phi\right\}}{\pi R_{\mathrm{eq}}^{2}\langle\delta T\rangle}$,

where $\psi$ is a suitably chosen phase, $\langle\delta T\rangle$ is given in Eq. (45), and $f(\theta)$ corresponds to the visibility curve (i.e. the border between the visible and hidden side of the star). The function $f$ obeys the following symmetry: $f(\theta)+f(\pi-\theta)=\pi$. We have made use of Eqs. (16) and (22) in obtaining an explicit expression for $\boldsymbol{e}_{\mathrm{obs}} \cdot \mathrm{d} \boldsymbol{S}$. The integral in Eq. (C.1) is then split into two equal halves and the second half is modified according to the variable changes $\theta^{\prime}=\pi-\theta$ and $\phi^{\prime}=\phi-\pi$ :

$$
\begin{aligned}
D(i) \cos (\omega t+\psi)= & \mathfrak{R}\left\{\int_{\theta=0}^{\pi} \int_{\phi=-f(\theta)}^{f(\theta)} \delta \hat{T}(\theta) \mathrm{e}^{\mathrm{i} m \phi+i \omega t}\left[r(\sin i \sin \theta \cos \phi+\cos i \cos \theta)-r_{\theta}(\sin i \cos \theta \cos \phi-\cos i \sin \theta)\right] r \sin \theta \mathrm{d} \theta \mathrm{d} \phi\right. \\
& +\int_{\theta^{\prime}=0}^{\pi} \int_{\phi^{\prime}=f\left(\theta^{\prime}\right)-2 \pi}^{-f\left(\theta^{\prime}\right)}(-1)^{m+1} \delta \hat{T}\left(\pi-\theta^{\prime}\right) \mathrm{e}^{\mathrm{i} m \phi^{\prime}+i \omega t}\left[r\left(\sin i \sin \theta^{\prime} \cos \phi^{\prime}+\cos i \cos \theta^{\prime}\right)\right. \\
& \left.\left.-r_{\theta^{\prime}}\left(\sin i \cos \theta^{\prime} \cos \phi^{\prime}-\cos i \sin \theta^{\prime}\right)\right] r \sin \theta^{\prime} \mathrm{d} \theta^{\prime} \mathrm{d} \phi^{\prime}\right\} /\left(2 \pi R_{\mathrm{eq}}^{2}\langle\delta T\rangle\right),
\end{aligned}
$$

where we have made use of the relation $f(\theta)+f(\pi-\theta)=\pi$. The two halves can be combined to give a single integral over the entire stellar surface only if $\delta \hat{T}(\theta)=(-1)^{m+1} \delta \hat{T}(\pi-\theta)$. If we assume this is the case, we can then see under conditions the integral vanishes. We specifically look at the integration over $\phi$, which now is between the bounds 0 and $2 \pi$. Remembering that $\int_{0}^{2 \pi} \mathrm{e}^{\mathrm{i} m \phi} \cos \phi \mathrm{d} \phi=0$ if $|m| \neq 1$ and that $\int_{0}^{2 \pi} \mathrm{e}^{\mathrm{i} m \phi} \mathrm{d} \phi=0$ if $m \neq 0$, we deduce the second condition for cancelling the disk-integration factor, i.e. $|m| \geq 2$.

\section{Appendix D: Normalisation of multi-colour visibilities}

In order to find a normalisation which minimises the distances between a set of multi-colour visibilities, we start with the cost function given in Eq. (47) and include an additional term so as to enforce the constraint $\sum_{i=1}^{b} W_{i}^{2}=1$. Without loss of generality, we work with the normalised components, $\tilde{V}_{i}^{j}$, instead of the original ones:

$J=\sum_{j=1}^{N} \sum_{i=1}^{b}\left(\tilde{A}_{j} \tilde{V}_{i}^{j}-W_{i}\right)^{2}+\Lambda\left(1-\sum_{i=1}^{b} W_{i}^{2}\right)$

where $\Lambda$ represents a Lagrange multiplier. Setting the derivatives, $\partial J / \partial \tilde{A}_{j}, \partial J / \partial W_{i}$, and $\partial J / \partial \Lambda$, to zero leads to the following system:

$$
\begin{aligned}
\tilde{A}_{j} & =\frac{\tilde{\boldsymbol{V}}^{j} \cdot \boldsymbol{W}}{\tilde{\boldsymbol{V}}^{j} \cdot \tilde{\boldsymbol{V}}^{j},} \\
(N-\Lambda) \boldsymbol{W} & =\sum_{j=1}^{N} \tilde{A}_{j} \tilde{\boldsymbol{V}}^{j}, \\
\boldsymbol{W} \cdot \boldsymbol{W} & =1,
\end{aligned}
$$

where we've used vectorial notation for conciseness and where $\boldsymbol{A} \cdot \boldsymbol{B}$ represents the dot product $\sum_{i=1}^{b} A_{i} B_{i}$. Using the normalised components $\tilde{V}_{i}^{j}$ allows us to simplify Eq. (D.2) to $\tilde{A}_{j}=\tilde{\boldsymbol{V}}^{j} \cdot \boldsymbol{W}$. When combined with Eq. (D.3), this yields:

$(N-\Lambda) \boldsymbol{W}=\sum_{j=1}^{N}\left(\tilde{\boldsymbol{V}}^{j} \cdot \boldsymbol{W}\right) \tilde{\boldsymbol{V}}^{j}$

This last equation is in fact an eigenvalue problem where $N-\Lambda$ is the eigenvalue and $\boldsymbol{W}$ the eigenvector. In order to determine which eigensolution yields the minimal value for $J$, we develop the cost function as follows:

$J=\sum_{j=1}^{N}\left\{\tilde{A}_{j}^{2} \tilde{\boldsymbol{V}}^{j} \cdot \tilde{\boldsymbol{V}}^{j}-2 \tilde{A}_{j} \tilde{\boldsymbol{V}}^{j} \cdot \boldsymbol{W}+\boldsymbol{W} \cdot \boldsymbol{W}\right\}=\sum_{j=1}^{N}\left\{1-\tilde{A}_{j}^{2}\right\}=N-\sum_{j=1}^{N} \tilde{A}_{j}^{2}$,

where we've used the simplifications $\tilde{\boldsymbol{V}}^{j} \cdot \tilde{\boldsymbol{V}}^{j}=1, \tilde{A}_{j}=\tilde{\boldsymbol{V}}^{j} \cdot \boldsymbol{W}$, and $\boldsymbol{W} \cdot \boldsymbol{W}=1$. In order to simplify the term $\sum_{j=1}^{N} \tilde{A}_{j}^{2}$, we calculate the dot product between $\boldsymbol{W}$ and Eq. (D.3):

$(N-\Lambda) \boldsymbol{W} \cdot \boldsymbol{W}=N-\Lambda=\sum_{j=1}^{N} \tilde{A}_{j}\left(\tilde{\boldsymbol{V}}^{j} \cdot \boldsymbol{W}\right)=\sum_{j=1}^{N} \tilde{A}_{j}^{2}$. 
Hence,

$J=N-(N-\Lambda)=\Lambda$.

Therefore, the minimal value of $\Lambda$ (and hence the maximal value of $N-\Lambda$ ) corresponds to the minimal value of $J$. The vector $W$ is therefore the principal component of the vector set $\left(\tilde{\boldsymbol{V}}^{j}\right)_{j=1 \ldots N}$ and can be found either via a singular value decomposition of the associated matrix, or more simply through a power iteration. 